\title{
An Information Theoretic Framework For Designing Information Elicitation Mechanisms That Reward Truth-telling
}

\author{
YUQING KONG, Peking University, China \\ GRANT SCHOENEBECK, University of Michigan, USA
}

In the setting where information cannot be verified, we propose a simple yet powerful information theoretical framework-the Mutual Information Paradigm-for information elicitation mechanisms. Our framework pays every agent a measure of mutual information between her signal and a peer's signal. We require that the mutual information measurement has the key property that any "data processing" on the two random variables will decrease the mutual information between them. We identify such information measures that generalize Shannon mutual information.

Our Mutual Information Paradigm overcomes the two main challenges in information elicitation without verification: (1) how to incentivize high-quality reports and avoid agents colluding to report random or identical responses; (2) how to motivate agents who believe they are in the minority to report truthfully.

Aided by the information measures, we found (1) we use the paradigm to design a family of novel mechanisms where truth-telling is a dominant strategy and pays better than any other strategy profile (in the multi-question, detail free, minimal setting where the number of questions is large); (2) we show the versatility of our framework by providing a unified theoretical understanding of existing mechanisms-Bayesian Truth Serum Prelec (2004) and Dasgupta and Ghosh (2013) - by mapping them into our framework such that theoretical results of those existing mechanisms can be reconstructed easily.

We also give an impossibility result that illustrates, in a certain sense, the the optimality of our framework.

CCS Concepts: • Theory of computation $\rightarrow$ Algorithmic mechanism design; • Mathematics of computing $\rightarrow$ Information theory;

Additional Key Words and Phrases: Peer prediction, information theory, crowdsourcing, mechanism design

ACM Reference format:

Yuqing Kong and Grant Schoenebeck. 2019. An Information Theoretic Framework For Designing Information Elicitation Mechanisms That Reward Truth-telling. ACM Trans. Econ. Comput. 7, 1, Article 2 (January 2019), 33 pages.

https://doi.org/10.1145/3296670

This work is supported by the National Science Foundation, under Grants CAREER\#1452915, CCF\#1618187, and AitF\#1535912.

Authors' addresses: Y. Kong, Peking University, The Center on Frontiers of Computing Studies, Courtyard No. 5, Jing Yuan, No. 5 Yiheyuan Road Haidian District, Beijing, P. R. China, 100871; email: yuqing.kong@pku.edu.cn; G. Schoenebeck, University of Michigan, School of Information, 4322 North Quad, 105 S. State St., Ann Arbor, MI 48109; email: schoeneb@ umich.edu.

Permission to make digital or hard copies of all or part of this work for personal or classroom use is granted without fee provided that copies are not made or distributed for profit or commercial advantage and that copies bear this notice and the full citation on the first page. Copyrights for components of this work owned by others than the author(s) must be honored. Abstracting with credit is permitted. To copy otherwise, or republish, to post on servers or to redistribute to lists, requires prior specific permission and/or a fee. Request permissions from permissions@acm.org.

(C) 2019 Copyright held by the owner/author(s). Publication rights licensed to ACM.

2167-8375/2019/01-ART2 \$15.00

https://doi.org/10.1145/3296670

ACM Transactions on Economics and Computation, Vol. 7, No. 1, Article 2. Publication date: January 2019. 


\section{INTRODUCTION}

User feedback requests (e.g., Ebay's reputation system and the innumerable survey requests in one's email inbox) are increasingly prominent and important. However, the overwhelming number of requests can lead to low participation rates, which in turn may yield unrepresentative samples. To encourage participation, a system can reward people for answering requests. But this may cause perverse incentives: some people may answer a large number of questions simply for the reward and without making any attempt to answer accurately. In this case, the reviews the system obtains may be inaccurate and meaningless. Moreover, people may be motivated to lie when they face a potential loss of privacy or can benefit in the future by lying now.

It is thus important to develop systems that motivate honesty. If we can verify the information people provide in the future (e.g., prediction markets), then we can motivate honesty via this future verification. However, sometimes we need to elicit information without verification, since the objective truth is hard to access or even does not exist (e.g., do you like this restaurant?). In our article, we focus on the situation where the objective truth is not observable.

Two main challenges in information elicitation without verification area are:

(1) How to incentivize high-quality reports and avoid colluding agents who report random or identical responses; and

(2) How to motivate agents who believe they are in the minority to report truthfully.

The typical solution desiderata to the above challenges are:

(strictly) truthful: truth-telling is a (strict) Bayesian Nash equilibrium.

informed truthful: ${ }^{1}$ the truth-telling equilibrium is paid more than other strategy profiles in expectation and strictly more than other uninformed strategy (e.g., always say yes) profiles.

strongly truthful: the truth-telling equilibrium is paid more than other strategy profiles in expectation and strictly more than other non-permutation strategy profiles. ${ }^{2}$

A weaker version of informed truthful/strongly truthful is to only compare truth-telling with other equilibria. We call this weaker version as informed truthful/strongly truthful with respect to all equilibria.

We additionally value mechanisms that are:

detail free: require no knowledge of the prior;

minimal: only require agents to report their information rather than forecasts for other agents' reports; and

dominantly truthful: truth-telling maximizes the expected payment regardless of the other agents' strategies and for every agent, if she believes at least one other agent will tell the truth, truth-telling pays her strictly better than playing a "non-permutation" strategy.

Two main settings are considered in the information elicitation without verification literature. In the single-question setting, each agent is asked a single question (e.g., Do you like this restaurant?) and is assumed to have a common prior, which means agents who receive the same signal (Y/N) have the same belief for the world. Miller et al. (2005) and Prelec (2004) are two seminal works in this setting. Another is the multi-question setting in which each agent is asked a batch of $a$ priori similar questions (e.g., is there a cat in this picture?). Dasgupta and Ghosh (2013) is the foundational work in this setting. Many works (Faltings et al. 2014; Prelec 2004; Radanovic and Faltings

\footnotetext{
${ }^{1}$ We borrow this terminology from Shnayder et al. (2016).

${ }^{2}$ The strategy where an agent permutes her private signal is defined as permutation strategy. Formal definition is given in Section 2
} 
2013, 2014; Riley 2014; Witkowski 2014; Witkowski and Parkes 2012; Zhang and Chen 2014) have successfully designed truthful and detail free mechanisms. The design of informed/strongly truthful and detail free mechanisms seems to be more complicated. While two prior works, Dasgupta and Ghosh (2013); Prelec (2004), have successfully designed (a weaker version of ${ }^{3}$ ) informed truthful and detail free mechanisms, their results are typically proved by clever algebraic computations and sometimes lack a deeper intuition and also fail to extend to important settings.

\subsection{Our Contributions}

To give a clear presentation, the current article only considers the setting where agents do not need any effort to receive the private signals. Our techniques can be also applied to the setting where we assume agents need to invest effort to obtain private signals (see Section 7 for more discussions).

The main contribution of the current article is to provide a simple yet powerful information theoretic paradigm-the Mutual Information Paradigm (Section 3)-for designing information elicitation mechanisms that are informed/strongly truthful, and, detail-free. Moreover, some of the mechanisms based on our paradigm are additionally minimal and dominantly truthful. To the best of our knowledge, ours is the first dominantly truthful information elicitation mechanism without verification, in the setting where agents do not need effort to receive their private information.

High Level Techniques and Insights: Our framework provides a key insight by distilling the essence of building information elicitation mechanisms to information theory properties. If we measure "information" correctly, then any non-truthful strategy will decrease the amount of "information" there exists between signals. Therefore, the mechanism should reward every agent according to the amount of information she provides. That is, the information elicitation mechanism should be "information-monotone."

To design "information-monotone" mechanisms, we find two families of "(weakly) informationmonotone" information measures- $f$-mutual information and Bregman mutual information-both of which generalize the Shannon mutual information.

Information theory is not typically used in the information elicitation literature, a key novelty of our work is showing how the insights of information theory illuminate the work and challenges in the information elicitation field.

Applications of Framework: Aided by the Mutual Information Paradigm,

(1) In the multi-question setting, we exhibit two families of novel mechanisms that are dominantly truthful, detail free, and minimal when the number of questions is largethe $f$-mutual information mechanism and the Bregman mutual information mechanism (Section 5.1). Additionally, the $f$-mutual information mechanism is also strongly truthful.

We also use a specific $f$-mutual information mechanism to map Dasgupta and Ghosh (2013), into our framework with an extra assumption they make and easily reconstruct their main results (Section 5.2), which apply to the binary choice case and do not require a large number of questions but also do not have truth-telling as a dominant strategy (only a Bayesian Nash equilibrium). We also map Shnayder et al. (2016) into our framework (Section 5.2.3).

(2) In the single-question setting, we show that the log scoring rule is an unbiased estimator of Shannon mutual information (Section 4.2). Aided by this observation, we map Prelec (2004) (Section 6.1) into our information theoretic framework and allow the easy

\footnotetext{
${ }^{3}$ Prelec (2004)'s mechanism is informed truthful with respect to all equilibria. Dasgupta and Ghosh (2013)'s mechanism is
} informed truthful. 
reconstruction of the theoretical results of Prelec (2004) (Section 6.1.2), which is a very important work in the information elicitation literature. We believe our framework highlights important insights of Prelec (2004), which can be extended to other settings.

Finally, we give impossibility results (Appendix A), which imply that no truthful detail-free mechanism can pay truth-telling strictly better than the permutation strategy in certain settings. This illustrates, in a certain sense, the optimality of our framework.

\subsection{Related Work}

Since Miller et al. (2005) introduced peer prediction, several works follow the peer prediction framework and design information elicitation mechanisms without verification in different settings. In this section, we introduce these work by classifying them into the following categories.

(1) Multi-question, Detail Free, Minimal Setting: Dasgupta and Ghosh (2013) consider a setting where agents are asked to answer multiple a priori similar binary choice questions. They propose a mechanism $M_{d}$ that pays each agent the correlation between her answer and her peer's answer, and show each agent obtains the highest payment if everyone tells the truth. In retrospect, one can see that our techniques are a recasting and generalization of those of Dasgupta and Ghosh (2013). Kamble et al. (2015) considers both homogeneous and heterogeneous populations and design a mechanism such that truth-telling pays higher than non-informative equilibria in the presence of a large number of a priori similar questions. However, they leave the analysis of other non-truthful equilibria as an open question. Agarwal et al. (2017) consider a peer prediction mechanism for heterogeneous users.

(2) Single-question, Detail free, Common Prior, Non-minimal Setting: Prelec (2004) proposes Bayesian Truth Serum (BTS) and the signal-prediction framework for the setting that agents are asked to answer only one question and the mechanism does not know the common prior. Prelec (2004) shows when the number of agents is infinite, the case everyone tells the truth is both an equilibrium and that the total payments agents receive in expectation is at least as high as in any other equilibria. Logarithmic Peer Truth Serum (PTS) (Radanovic and Faltings 2015) extends BTS to a slightly different setting involving sensors, but still requires a large number of agents.

The biggest limitation of Prelec (2004) is that the number of agents is assumed to be infinite even to make truth-telling an equilibrium. Faltings et al. (2014); Radanovic and Faltings (2013, 2014); Riley (2014); Witkowski (2014); Witkowski and Parkes (2012); Zhang and Chen (2014) successfully weaken this assumption. However, all of these works lack the analysis of non-truthful equilibria. In contrast, the disagreement mechanism designed by Kong and Schoenebeck (2018a) is truthful and pays truth-telling strictly better than any other symmetric equilibrium if the number of agents is greater than 6 .

(3) Single-question, Known Common Prior, Minimal Setting: Jurca and Faltings $(2007,2009)$ use algorithmic mechanism design to build their own peer prediction style mechanism where truthtelling is paid strictly better than non-truthful pure strategies but leave the analysis of mixed strategies as an open question. Frongillo and Witkowski (2016) consider the design for robust, truthful and minimal peer prediction mechanisms with the prior knowledge and lack the analysis of nontruthful equilibria. Kong et al. (2016) modify the peer prediction mechanism such that truth-telling is paid strictly better than any other non-truthful equilibrium. Additionally, they optimize the difference between the truth-telling equilibrium and the next best paying informative equilibrium. However, the mechanism still needs to know the prior and the analysis only works for the case of binary signals.

(4) Expertise elicitation: Gao, Wright, and Leyton-Brown (Gao et al. 2016) show that all traditional peer-prediction mechanisms, generally fail in motivating the agents to invest effort to report the desired signal when spurious "cheap signals" exist. For example, when peer grading an essay, peers 
could instead simply report the grammatical correctness of the essay. Spot-checking the grammar would presumably require much less effort than evaluating the ideas of the essay. However, it would still allow agents to correlate on a signal, just not the desired signal, and thus be rewarded by a peer-prediction mechanism. In general, cheap signals can ensure agreement and may even be correlated with the sought signal.

This work considers a model where each agent receives a single private signal, and so no cheap signals exist. A subsequent work (Kong and Schoenebeck 2018b) of the current article deals with a model that can include cheap signal by making an additional assumption, more sophisticated agents know the beliefs of less sophisticated agents, and combines this assumption with the current article's framework to propose mechanisms that address the issue raised by Gao, Wright, and Leyton-Brown (Gao et al. 2016).

(5) Other models: Liu and Chen (2017) design peer prediction mechanism in the machine learning setting. Mandal et al. (2016) consider peer prediction mechanism for heterogeneous tasks. Witkowski et al. (2017) consider the setting where the mechanism elicits the forecasts from the crowds. Witkowski et al. (2017) incentivize high-quality forecasts report by only assuming the knowledge of the unbiased estimator of the optimal forecast rather than the (future) verification of the forecasts (e.g., prediction market). A subsequent work (Kong and Schoenebeck 2018c) of the current article shows a way to remove Witkowski et al. (2017)'s assumption by following the current article's framework.

1.2.1 Independent Work and Subsequent Work. An earlier version of this article was posted to arXiv in 2016 (Kong and Schoenebeck 2016). The current version augments the original version in two ways: the current article adds Section 4.2 and an analysis of the independent work, Shnayder et al. (2016), in the Mutual Information Paradigm framework.

Independent Work. Like this article's earlier version, Shnayder et al. (2016) also extends Dasgupta and Ghosh (2013)'s binary signals mechanism to multiple signals setting. However, the two works differ both in the specific mechanism and the technical tools employed.

Shnayder et al. (2016) analyze how many questions are needed (whereas we simply assume infinitely many questions). Like our article, they also analyze to what extent truth-telling can pay strictly more than other equilibria. Additionally, they show their mechanism does not need a large number of questions when "the signal correlation structure" is known (that is the pair-wise correlation between the answers of two questions). While Kong and Schoenebeck (2016) does not state such results, we note that the techniques employed are sufficiently powerful to immediately extend to this interesting special case-when the signal structure is known, it is possible to construct an unbiased estimator for $f$-mutual information of the distribution, when the total variation distance is used to define the $f$-mutual information. The current article shows the analysis for this in Section 5.2.3. Both articles also show their results generalize Dasgupta and Ghosh (2013).

Moreover, when the number of questions is large, the $f$-mutual information mechanism has truth-telling as a dominant strategy while Shnayder et al. (2016) do not.

Subsequent Work. Liu and Chen (2018) also achieve truthful elicitation in dominant strategy in a work subsequent to ours. Their work analyzes the sample complexity of their mechanism and shows that it does not require an infinite number of tasks, but their mechanism is restricted to the binary choice setting.

\section{PRELIMINARIES}

General Setting. We introduce the general setting $(n, \Sigma)$ of the mechanism design framework where $n$ is the number of agents and $\Sigma$ is the set of all possible private signals. Each agent $i$ receives a 
random private information/signal $\Psi_{i}: \Omega \mapsto \Sigma$, where $\Omega$ is the underlying sample space. She also has a prior for other agents' private information.

Formally, each agent $i$ believes the agents' private information is chosen from a joint distribution $Q_{i}$ before she receives her private information. Thus, from agent $i$ 's perspective, before she receives any private information, the probability that agent 1 receives $\Psi_{1}=\sigma_{1}$, agent 2 receives $\Psi_{2}=\sigma_{2}, \ldots$, agent $n$ receives $\Psi_{n}=\sigma_{n}$ is $Q_{i}\left(\Psi_{1}=\sigma_{1}, \Psi_{2}=\sigma_{2}, \ldots, \Psi_{n}=\sigma_{n}\right)$. After she receives her private information based on her prior, agent $i$ will also update her knowledge to a posterior distribution, which is the prior conditioned on her private information. Without assuming a common prior, agents may have different priors, that is, $Q_{i}$ may not equal $Q_{j}$. We define $\Delta_{\Sigma}$ as the set of all possible probability distributions over $\Sigma$.

In some situations (e.g., restaurant reviews), agents do not need any effort to receive the private signals when they answer the questions, while in other situations (e.g., peer reviews), agents need to invest a certain amount of effort to receive the private signals. To give a clear presentation, the current article only considers the setting where agents do not need any effort to receive the private signals. Our techniques can be also applied to the setting where we assume agents need to invest effort to obtain private signals (see Section 7 for more discussions).

\subsection{Basic Game Theory Concepts}

Definition 2.1 (Mechanism). We define a mechanism $\mathcal{M}$ for a setting $(n, \Sigma)$ as a tuple $\mathcal{M}:=(\mathcal{R}, M)$ where $\mathcal{R}$ is a set of all possible reports the mechanism allows, and $M: \mathcal{R}^{n} \mapsto \mathbb{R}^{n}$ is a mapping from all agents' reports to each agent's reward.

The mechanism requires agents to submit a report $r$. For example, $r$ can simply be an agent's private information. In this case, $\mathcal{R}=\Sigma$. We call this kind of mechanism a minimal mechanism. We define $\mathbf{r}$ to be a report profile $\left(r_{1}, r_{2}, \ldots, r_{n}\right)$ where $r_{i}$ is agent $i$ 's report.

Typically, the strategy of each agent should be a mapping from her received knowledge including her prior and her private signal, to a probability distribution over her report space $\mathcal{R}$. But since all agents' priors are fixed during the time when they play the mechanism, without loss of generality, we omit the prior in the definition of strategy.

Definition 2.2 (Strategy). Given a mechanism $\mathcal{M}$, we define the strategy of each agent in the mechanism $\mathcal{M}$ for setting $(n, \Sigma)$ as a mapping $s$ from $\sigma$ (private signal) to a probability distribution over $\mathcal{R}$.

We define a strategy profile $\mathbf{s}$ as a profile of all agents' strategies $\left(s_{1}, s_{2}, \ldots, s_{n}\right)$, and we say agents play $\mathbf{s}$ if for all $i$, and agent $i$ plays strategy $s_{i}$.

Note that actually the definition of a strategy profile only depends on the setting and the definition of all possible reports $\mathcal{R}$. We will need the definition of a mechanism when we define an equilibrium.

A Bayesian Nash equilibrium consists of a strategy profile $s=\left(s_{1}, \ldots, s_{n}\right)$ such that no agent wishes to change her strategy, since other strategies will decrease her expected payment, given the strategies of the other agents and the information contained in her prior and her signal.

Definition 2.3 (Agent Welfare). Given a mechanism $\mathcal{M}$, for a strategy profile s, we define the agent welfare of $\mathbf{s}$ as the sum of expected payments to agents when they play s under $\mathcal{M}$.

Transition Probability. We define a $m \times m^{\prime}$ transition matrix $M \in \mathbb{R}^{m \times m^{\prime}}$ as a matrix such that for any $i, j \in[m] \times\left[m^{\prime}\right], M_{i, j} \geq 0$ and $\sum_{j} M_{i, j}=1$. We define a permutation transition matrix $\pi$ as a $m \times m$ permutation matrix.

Given a random variable $X$ with $m$ possible outcomes, by abusing notation a little bit, a $m \times m^{\prime}$ transition matrix $M$ defines a transition probability $M$ that transforms $X$ to $M(X)$ such that 
$X^{\prime}:=M(X)$ is a new random variable that has $m^{\prime}$ possible outcomes where $\operatorname{Pr}\left[X^{\prime}=j \mid X=i\right]=$ $M_{i, j}$.

If the distribution of $X$ is represented by an $m \times 1$ column vector $\mathbf{p}$, then the distribution over $M(X)$ is $M^{T} \mathbf{p}$ where $M^{T}$ is the transpose of $M$.

We can use transition matrices to represent agents' strategies of reporting their private information. Given the general setting $(n, \Sigma)$, for the minimal mechanisms, fixing the priors of the agents, each agent $i$ 's strategy $s_{i}$ can be seen as a transition matrix that transforms her private information $\Psi_{i}$ to her reported information $\hat{\Psi}_{i}=s_{i}\left(\Psi_{i}\right)$. We define truth-telling $\mathrm{T}$ as the strategy where an agent truthfully reports her private signal. T corresponds to an identity transition matrix.

We say agent $i$ plays a permutation strategy if $s_{i}$ corresponds to a permutation transition matrix. An example is that an agent relabels/permutes the signals and reports the permuted version (e.g., she reports "good" when her private signal is "bad" and reports "bad" when her private signal is "good"). Note that $\mathrm{T}^{4}$ is a permutation strategy as well. We call the strategy profile where all agents play a permutation strategy a permutation strategy profile. Note that in a permutation strategy profile, agents may play different permutation strategies. When a permutation strategy profile is a Bayesian Nash equilibrium, we call such equilibrium a permutation equilibrium.

An uninformed strategy has the same report distribution for all signals. Note that the strategy that agent $i$ always reports the same signal (e.g., always say yes) is an uninformed strategy. The strategy that agent $i$ ignores her private signal and reports a random coin flip's output is an uninformed strategy as well. We call the strategy profile where all agents play an uninformed strategy a uninformed strategy profile.

\subsection{Mechanism Design Goals}

We hope our mechanisms can be strictly truthful, and even informed truthful, strongly truthful and dominantly truthful (see informal definitions in Section 1 and formal definitions will be introduced later). In addition to the above equilibrium goals, we also hope the mechanism can be minimal and detail free (see definitions in Section 1).

It turns out no truthful detail free mechanism can make truth-telling strategy profile be strictly better than any permutation strategy profile (Section A). Therefore, the best we can hope is making the truth-telling strategy profile be strictly better than any other non-permutation strategy profile. We give the formal definitions for the equilibrium goals here.

Mechanism Design Goals.

(Strictly) truthful A mechanism $\mathcal{M}$ is (strictly) truthful if for every agent, $\mathrm{T}$ (uniquely) maximizes her expected payment given that everyone else plays $\mathrm{T}$.

Dominantly truthful A mechanism $\mathcal{M}$ is dominantly truthful if for every agent, $\mathrm{T}$ maximizes her expected payment no matter what strategies other agents play; and for every agent, if she believes at least one other agent will tell the truth, playing $\mathrm{T}$ pays her strictly higher than playing a non-permutation strategy.

Strongly truthful A mechanism $\mathcal{M}$ is strongly truthful if it is truthful and the truth-telling equilibrium maximizes the agent welfare among all strategy profiles and any other nonpermutation strategy profile has strictly less agent welfare.

Informed truthful A mechanism $\mathcal{M}$ is informed truthful if it is truthful, the truth-telling equilibrium maximizes the agent welfare among all strategy profiles, and any other uninformed strategy profile has strictly less agent welfare.

\footnotetext{
${ }^{4}$ The above definitions of $\mathrm{T}$ and the permutation strategy are sufficient to analyze the general framework. When considering more specific settings, we will provide specific definitions for those settings.
} 
Additionally, we say a mechanism is strongly truthful/informed truthful with respect to all equilibria if the truth-telling equilibrium maximizes the agent welfare among all equilibria rather than all strategy profiles. This restricted property is usually considered in the single-question setting.

Strongly truthful $>$ informed truthful $>$ truthful; dominantly truthful $>$ truthful. The strongly truthful property is incomparable with the dominantly truthful property. In the later section, we will see a dominantly truthful mechanism may not be strongly truthful (e.g., Bregman mutual information mechanism in Section 5.1).

\subsection{Mechanism Design Tools}

$f$-divergence (Ali and Silvey 1966; Csiszár et al. 2004). $f$-divergence $D_{f}: \Delta_{\Sigma} \times \Delta_{\Sigma} \rightarrow \mathbb{R}$ is a nonsymmetric measure of the difference between distribution $\mathbf{p} \in \Delta_{\Sigma}$ and distribution $\mathbf{q} \in \Delta_{\Sigma}$ and is defined to be

$$
D_{f}(\mathbf{p}, \mathbf{q})=\sum_{\sigma \in \Sigma} \mathbf{p}(\sigma) f\left(\frac{\mathbf{q}(\sigma)}{\mathbf{p}(\sigma)}\right),
$$

where $f(\cdot)$ is a convex function and $f(1)=0$. Now we introduce the properties of $f$-divergence:

Fact 2.4 (Non-Negativity (Csiszár et Al. 2004)). For any p, $\mathbf{q}, D_{f}(\mathbf{p}, \mathbf{q}) \geq 0$ and $D_{f}(\mathbf{p}, \mathbf{q})=0$ if and only if $\mathbf{p}=\mathbf{q}$.

Fact 2.5 (Joint Convexity (Csiszár et al. 2004)). For any $0 \leq \lambda \leq 1$, for any $\mathbf{p}_{1}, \mathbf{p}_{2}, \mathbf{q}_{1}, \mathbf{q}_{2} \in \Delta_{\Sigma}$,

$$
D_{f}\left(\lambda \mathbf{p}_{1}+(1-\lambda) \mathbf{p}_{2}, \lambda \mathbf{q}_{1}+(1-\lambda) \mathbf{q}_{2}\right) \leq \lambda D_{f}\left(\mathbf{p}_{1}, \mathbf{q}_{1}\right)+(1-\lambda) D_{f}\left(\mathbf{p}_{2}, \mathbf{q}_{2}\right) .
$$

Fact 2.6 (Information Monotonicity ((Ali and Silvey 1966; Amari And Cichocki 2010; Liese AND VAJDA 2006))). For any strictly convex function $f$, $f$-divergence $D_{f}(\mathbf{p}, \mathbf{q})$ satisfies information monotonicity so that for any transition matrix $\theta \in \mathbb{R}^{|\Sigma| \times|\Sigma|}, D_{f}(\mathbf{p}, \mathbf{q}) \geq D_{f}\left(\theta^{T} \mathbf{p}, \theta^{T} \mathbf{q}\right)$.

Moreover, the inequality is strict if and only if there exists $\sigma, \sigma^{\prime}, \sigma^{\prime \prime}$, such that $\frac{\mathbf{p}\left(\sigma^{\prime \prime}\right)}{\mathbf{p}\left(\sigma^{\prime}\right)} \neq \frac{\mathbf{q}\left(\sigma^{\prime \prime}\right)}{\mathbf{q}\left(\sigma^{\prime}\right)}$ and $\theta_{\sigma^{\prime}, \sigma} \mathbf{p}\left(\sigma^{\prime}\right)>0, \theta_{\sigma^{\prime \prime}, \sigma} \mathbf{p}\left(\sigma^{\prime \prime}\right)>0$.

The proof is in the Appendix for reference.

Definition 2.7. Given two signals $\sigma^{\prime}, \sigma^{\prime \prime} \in \Sigma$, we say two probability measures $\mathbf{p}$, q over $\Sigma$ can distinguish $\sigma^{\prime}, \sigma^{\prime \prime} \in \sum$ if $\mathbf{p}\left(\sigma^{\prime}\right)>0, \mathbf{p}\left(\sigma^{\prime \prime}\right)>0$ and $\frac{\mathbf{q}\left(\sigma^{\prime}\right)}{\mathbf{p}\left(\sigma^{\prime}\right)} \neq \frac{\mathbf{q}\left(\sigma^{\prime \prime}\right)}{\mathbf{p}\left(\sigma^{\prime \prime}\right)}$

Fact 2.6 directly implies

COROLlary 2.8. Given a transition matrix $\theta$ and two probability measures $\mathbf{p}, \mathbf{q}$ that can distinguish $\sigma^{\prime}, \sigma^{\prime \prime} \in \Sigma$, if there exists $\sigma \in \Sigma$, such that $\theta\left(\sigma^{\prime}, \sigma\right), \theta\left(\sigma^{\prime \prime}, \sigma\right)>0$, we have $D_{f}(\mathbf{p}, \mathbf{q})>D_{f}\left(\theta^{T} \mathbf{p}, \theta^{T} \mathbf{q}\right)$ when $f$ is a strictly convex function.

Now we introduce two $f$-divergences in common use: KL Divergence and Total Variation Distance.

Example 2.9 (KL Divergence). Choosing - $\log (x)$ as the convex function $f(x), f$-divergence becomes KL divergence $D_{K L}(\mathbf{p}, \mathbf{q})=\sum_{\sigma} \mathbf{p}(\sigma) \log \frac{\mathbf{p}(\sigma)}{\mathbf{q}(\sigma)}$

Example 2.10 (Total Variation Distance). Choosing $|x-1|$ as the convex function $f(x), f$ divergence becomes Total Variation Distance $D_{t v d}(\mathbf{p}, \mathbf{q})=\sum_{\sigma}|\mathbf{p}(\sigma)-\mathbf{q}(\sigma)|$

Proper Scoring Rules (Gneiting and Raftery 2007; Winkler 1969). A scoring rule PS: $\Sigma \times \Delta_{\Sigma} \rightarrow \mathbb{R}$ takes in a signal $\sigma \in \Sigma$ and a distribution over signals $\mathbf{p} \in \Delta_{\Sigma}$ and outputs a real number. A scoring 
rule is proper if, whenever the first input is drawn from a distribution $\mathbf{p}$, then $\mathbf{p}$ will maximize the expectation of $P S$ over all possible inputs in $\Delta_{\Sigma}$ to the second coordinate. A scoring rule is called strictly proper if this maximum is unique. We will assume throughout that the scoring rules we use are strictly proper. Slightly abusing notation, we can extend a scoring rule to be $P S: \Delta_{\Sigma} \times \Delta_{\Sigma} \rightarrow \mathbb{R}$ by simply taking $P S(\mathbf{p}, \mathbf{q})=\mathbb{E}_{\sigma \leftarrow \mathbf{p}}(\sigma, \mathbf{q})$. We note that this means that any proper scoring rule is linear in the first term.

Example 2.11 (Log Scoring Rule (Gneiting and Raftery 2007; Winkler 1969)). Fix an outcome space $\Sigma$ for a signal $\sigma$. Let $\mathbf{q} \in \Delta_{\Sigma}$ be a reported distribution. The Logarithmic Scoring Rule maps a signal and reported distribution to a payoff as follows:

$$
L(\sigma, \mathbf{q})=\log (\mathbf{q}(\sigma)) .
$$

Let the signal $\sigma$ be drawn from some random process with distribution $\mathbf{p} \in \Delta_{\Sigma}$.

Then the expected payoff of the Logarithmic Scoring Rule:

$$
\mathbb{E}_{\sigma \leftarrow \mathbf{p}}[L(\sigma, \mathbf{q})]=\sum_{\sigma} \mathbf{p}(\sigma) \log \mathbf{q}(\sigma)=L(\mathbf{p}, \mathbf{q}) .
$$

This value will be maximized if and only if $q=p$.

\section{AN INFORMATION THEORETIC MECHANISM DESIGN FRAMEWORK}

The original idea of peer prediction (Miller et al. 2005) is based on a clever insight: every agent's information is related to her peers' information and therefore can be checked using her peers' information. Inspired by this, we propose a natural yet powerful information theoretic mechanism design idea-paying every agent the "mutual information" between her reported information and her peer's reported information where the "mutual information" should be information-monotoneany "data processing" on the two random variables will decrease the "mutual information" between them.

Definition 3.1 (Information-Monotone Mutual Information). We say MI is information-monotone if and only if for any random variables $X: \Omega \mapsto \Sigma_{X}$ and $Y: \Omega \mapsto \Sigma_{Y}$ :

Symmetry $M I(X ; Y)=M I(Y ; X)$;

Non-negativity $M I(X ; Y)$ is always non-negative and is 0 if $X$ is independent with $Y$;

Data-processing inequality for any transition probability $M \in \mathbb{R}^{\left|\Sigma_{X}\right| \times\left|\Sigma_{X}\right|}$, when $Y$ is independent with $M(X)$ conditioning on $X, M I(M(X) ; Y) \leq M I(X ; Y)$.

We say $M I$ is strictly information-monotone with respect to a probability measure $P \in \Delta_{\Sigma_{X} \times \Sigma_{Y}}$ if when the joint distribution over $X$ and $Y$ is $P$, for any non-permutation $M$, when $Y$ is independent with $M(X)$ conditioning on $X, M I(M(X) ; Y)<M I(X ; Y)$.

Definition 3.2 (Conditional Mutual Information). Given three random variables $X, Y, Z$, we define $M I(X ; Y \mid Z)$ as

$$
\sum_{z} \operatorname{Pr}[Z=z] M I(X ; Y \mid Z=z)
$$

where $M I(X ; Y \mid Z=z):=\operatorname{MI}\left(X^{\prime} ; Y^{\prime}\right)$ where $\operatorname{Pr}\left[X^{\prime}=x, Y^{\prime}=y\right]=\operatorname{Pr}[X=x, Y=y \mid Z=z]$.

We now provide a paradigm for designing information elicitation mechanisms-the Mutual Information Paradigm. We warn the reader that this paradigm represents some "wishful thinking" in that is it clear the paradigm cannot compute the payments given the reports. We will resolve this "wishful thinking" when we apply this framework to various settings (see Section 3.1).

Mutual Information Paradigm (MIP(MI)). Given a general setting $(n, \Sigma)$, 
Report For each agent $i$, she is asked to provide her private information $\Psi_{i}$. We denote the actual information she reports as $\hat{\Psi}_{i}$. Given an agent's strategy, $\hat{\Psi}_{i}$ can be seen as a random variable, which depends both on the randomness of the prior and the randomness of the strategy.

Payment/Information Score We uniformly randomly pick a reference agent $j \neq i$ and denote his report as $\hat{\Psi}_{j}$. Agent $i$ is paid by her information score

$$
\operatorname{MI}\left(\hat{\Psi}_{i} ; \hat{\Psi}_{j}\right)
$$

where $M I$ is information-monotone.

Given a general setting $(n, \Sigma)$, we say $M I$ is strictly information-monotone with respect to prior $Q$ if for every pair $i, j, M I$ is strictly information-monotone with respect to $Q\left(\Psi_{i}, \Psi_{j}\right)$.

THEOREM 3.3. Given a general setting $(n, \Sigma)$, when MI is strictly information-monotone with respect to every agent's prior, the Mutual Information Paradigm MIP(MI) is dominantly truthful and strongly truthful.

Theorem 3.3 almost immediately follows from the data-processing inequality of the mutual information. The key observation in the proof is that applying any strategy to the information is essentially data processing and thus erodes information.

The next section will introduce two families of information measures: $f$-mutual information $M I^{f}$ and Bregman mutual information $B M I^{P S} . M I^{f}$ is information-monotone and $B M I^{P S}$ is "weakly" information-monotone (quasi-information-monotone) in the sense that it only satisfies the data-processing inequality in one of the two coordinates. $M I^{f}$ is strictly information monotone with respect to a large family of priors (see Definition 4.6 for fine-grained prior) and $B M I^{P S}$ is also strictly quasi-information-monotone with respect to a large family of priors. This property is sufficiently good, since with a bad prior when all agents' private signals are independent, no mutual information measurement can be strictly information-monotone, since there is no "mutual information" between the agents even when they are honest.

Note that the Mutual Information Paradigm is a conceptual/“wishful thinking” mechanism, since it requires the mechanism to be able to compute $M I\left(\hat{\Psi}_{i} ; \hat{\Psi}_{j}\right)$. However, naively computing this requires not only knowledge of the full joint distribution over all agents' random private information but also knowing the strategies employed by agents. To apply the paradigm in practice, a key observation is that if we design mechanisms such that the payment in the mechanism is an unbiased estimator of the payment in Mutual Information Paradigm, the designed mechanisms will obtain the desirable properties immediately according to Theorem 3.3. Section 3.1 will show a overview of how to design such mechanisms in both the multi-question and single-question settings without knowledge of the full joint distribution (nor wishful thinking).

Proof. For each agent $i$, for any strategy $s_{i}$ she plays, comparing with the case she honestly reports $\Psi_{i}$, her expected information score is

$$
\sum_{j \neq i} \frac{1}{n-1} M I\left(\hat{\Psi}_{i} ; \hat{\Psi}_{j}\right)=\sum_{j \neq i} \frac{1}{n-1} M I\left(s_{i}\left(\Psi_{i}\right) ; \hat{\Psi}_{j}\right) \leq \sum_{j \neq i} \frac{1}{n-1} M I\left(\Psi_{i} ; \hat{\Psi}_{j}\right),
$$

since $M I$ is information-monotone. Thus, in MIP $(M I)$, truth-telling maximizes the expected payment regardless of other strategies when $M I$ is information-monotone.

For the strictness guarantee, we need to show when agent $i$ believes at least one agent tells the truth, for agent $i$, any non-permutation strategy will strictly decrease her expected payment. Let us assume that agent $i$ believes agent $j_{0} \neq i$ plays $\mathrm{T}$. When $M I$ is strictly information-monotone with respect to every agent's prior, $M I$ is strictly information-monotone with respect to $Q_{i}\left(\Psi_{i}, \Psi_{j_{0}}\right)$ as 
well. Then the inequality of the above formula is strict if agent $i$ plays a non-permutation strategy $s_{i}$, since $M I\left(s_{i}\left(\Psi_{i}\right) ; \hat{\Psi}_{j_{0}}\right)=M I\left(s_{i}\left(\Psi_{i}\right) ; \Psi_{j_{0}}\right)<M I\left(\Psi_{i}, \Psi_{j_{0}}\right)$.

Thus, when $M I$ is strictly information-monotone with respect to every agent's prior, $\operatorname{MIP}(M I)$ is dominantly truthful.

Fixing other agents' strategies except agent $k$, for $i \neq k$, agent $i$ 's expected payment is

$$
\begin{aligned}
\sum_{j \neq i} \frac{1}{n-1} M I\left(\hat{\Psi}_{i} ; \hat{\Psi}_{j}\right) & =\sum_{j \neq i, k} \frac{1}{n-1} M I\left(\hat{\Psi}_{i} ; \hat{\Psi}_{j}\right)+\frac{1}{n-1} M I\left(\hat{\Psi}_{i} ; \hat{\Psi}_{k}\right) \\
& \leq \sum_{j \neq i, k} \frac{1}{n-1} M I\left(\hat{\Psi}_{i} ; \hat{\Psi}_{j}\right)+\frac{1}{n-1} M I\left(\hat{\Psi}_{i} ; \Psi_{k}\right) .
\end{aligned}
$$

Thus, agent $i$ 's expected payment decreases when truthful agent $k$ changes to play a non-truthful strategy. For $i=k$, the dominantly truthful property already shows agent $i=k$ 's expected payment will decrease when truthful agent $k$ changes to play a non-truthful strategy. Therefore when $M I$ is information-monotone, in MIP $(M I)$, truth-telling strategy profile maximizes the agent welfare.

For the strictness guarantee, when $M I$ is strictly information-monotone with respect to every agent's prior, if truthful agent $k$ changes to play a non-permutation strategy $s_{k}$, then a truthful agent $i$ 's expected payment will strictly decrease, since $M I\left(\Psi_{i} ; s_{k}\left(\Psi_{k}\right)\right)<M I\left(\Psi_{i} ; \Psi_{k}\right)$ if $s_{k}$ is a nonpermutation strategy and $M I$ is strictly information-monotone.

Therefore, when $M I$ is strictly information-monotone with respect to every agent's prior, $\mathrm{MIP}(M I)$ is strongly truthful.

\subsection{Applying the Information Theoretic Framework: An Overview}

Directly calculating the information measure in our framework MIP requires knowledge of the joint distribution over agents' reported signals, which is unrealistic in practice. To resolve this "wishful thinking," a key observation is that paying agents an unbiased estimator of the information measure is sufficient when we assume agents are expected utility maximizers. To construct an unbiased estimator of the information measure using agents' reports, different settings have different techniques. In the multi-question setting, either we ask a large number of questions to estimate the information measure (see the $f$-mutual information mechanism and the Bregman mutual information mechanism in Section 5.1), or we ask a small number questions but require knowledge of the correlation structure (e.g., positively correlated/negatively correlated, see Section 5.2.3 for formal definition) and use a special $f$-mutual information (see the following paragraphs and the mapping of Dasgupta and Ghosh (2013) and Correlated Agreement mechanism (Shnayder et al. 2016) in Section 5.2 for more details). In the single-task setting, we ask agents their posteriors (e.g., what percentage of your peers say yes?) and construct the estimator using both the first order information (e.g., Y/N) and the second order information (e.g., 80\% Yes) (see the mapping of Bayesian Truth Serum (Prelec 2004) in Section 6.1). Thus, we can apply the MIP to design detail free mechanisms, which do not need any a priori knowledge of the distributions (nor wishful thinking).

$f$-mutual/Bregman Mutual Information Mechanism. Without loss of generality, we assume there are two agents, Alice and Bob. When we assign both Alice and Bob a large number of i.i.d. tasks, we will have a large number samples for Alice and Bob's joint reported signals $\left(\hat{\Psi}_{A} ; \hat{\Psi}_{B}\right)$, which allows us to estimate the $f$-mutual/Bregman mutual information and pay them this estimation. This is the idea of the $f$-mutual and Bregman mutual information mechanisms.

We will show that when agents are honest, the $f$-mutual information mechanism pays Alice and Bob

$$
M I^{f}\left(\Psi_{A} ; \Psi_{B}\right)
$$


and the Bregman mutual information mechanism pays Alice and Bob

$$
B M I^{P S}\left(\Psi_{A} ; \Psi_{B}\right)
$$

When agents are dishonest, the $f$-mutual information mechanism pays Alice and Bob $M I^{f}\left(\hat{\Psi}_{A} ; \hat{\Psi}_{B}\right)$ and the Bregman mutual information paradigm pays Alice and Bob $B M I^{P S}\left(\hat{\Psi}_{A} ; \hat{\Psi}_{B}\right)$.

We will show the $f$-mutual information mechanism is both dominantly truthful and strongly truthful via the direct application of Theorem 3.3 and the fact that $f$-mutual information is information monotone (Section 4). For the Bregman mutual information mechanism, since the Bregman mutual information only satisfies information monotonicity for one of the two coordinates, the Bregman mutual information mechanism is dominantly truthful but not necessarily strongly truthful, since an agent's lie may benefit other people.

TVD Mutual Information Mechanism. Without loss of generality, we assume there are two agents, Alice and Bob. We will show that essentially both Dasgupta and Ghosh (2013) and the Correlated Agreement mechanism (Shnayder et al. 2016) pay Alice and Bob a special $f$-mutual information, TVD mutual information (Example 4.3), between her information and her peer's information,

$$
M I^{t v d}\left(\Psi_{A} ; \Psi_{B}\right)
$$

when they are honest and pays Alice and Bob at most $M I^{t v d}\left(\hat{\Psi}_{A} ; \hat{\Psi}_{B}\right)$ when at least one of them is dishonest. Thus, Dasgupta and Ghosh (2013) and the Correlated Agreement mechanism (Shnayder et al. 2016) can be understood as a construction of an unbiased estimator of the TVD mutual information. To give a flavor of their mechanisms and the unbiased estimator of the TVD mutual information, we give an informal description here: both Dasgupta and Ghosh (2013) and the Correlated Agreement mechanism (Shnayder et al. 2016) can be interpreted as paying agents the average agreements for the same questions minusthe average agreements for different questions.

We use peer grading as the running example. Alice and Bob are both asked to grade the same three essays. When both Alice and Bob's answers are $(0,1,1)$, their average agreement for the same question is 1 and their average agreement for different questions is $2 / 6 .^{5}$ Thus, they will be paid $1-2 / 6=2 / 3$. This example also shows the difference between an $f$-mutual information style payment and a naive agreement payment. The $f$-mutual information style payment not only rewards agreement but also the diversity of the answers.

Bayesian Truth Serum. Finally, we show that agent $i$ 's information score of Bayesian Truth Serum(Prelec 2004) can be interpreted as a special $f$-mutual information, Shannon mutual information, between her information $\Psi_{i}$ and the state of the world $W$ conditioning on agent $j$ 's information $\Psi_{j}$,

$$
I\left(W ; \Psi_{i} \mid \Psi_{j}\right)
$$

when agents are honest and is less in other equilibria due to fact that the Shannon mutual information is information-monotone. Combining with the fact that the agent welfare in Bayesian Truth Serum is proportional to the sum of agents' information scores, the proof of the informed truthful property of the Bayesian truth serum can be reconstructed easily.

After interpreting the mechanisms by our information theoretic framework MIP, the "truth rewarding" properties of these mechanisms are naturally implied by the information-monotone property of MIP, which shows the versatility of our framework.

\footnotetext{
${ }^{5}$ Among all six question pairs $(k, l)$ such that $k \neq l$, they only agree for question pairs $(2,3)$ and $(3,2)$.
} 


\section{F-MUTUAL INFORMATION AND BREGMAN MUTUAL INFORMATION}

This section will introduce two information measures. $f$-mutual information is a family of information measures that are based on $f$-divergence while Bregman mutual information is a family of information measures that are based on Bregman divergence. $f$-mutual information is information-monotone. Bregman mutual information is strictly weaker than $f$-mutual information, since it only satisfies the information-monotonicity in one of its two coordinates and is asymmetric.

Plugging $f$-mutual information into MIP achieves dominantly truthful and strongly truthful mechanisms, while plugging Bregman mutual information into MIP only achieves dominantly truthful mechanisms, since when it only satisfies the information-monotonicity in one of its two coordinates, an agent's lie may benefit other agents.

\section{1 $f$-mutual Information}

Given two random variables $X, Y$, let $\mathbf{U}_{X, Y}$ and $\mathbf{V}_{X, Y}$ be two probability measures where $\mathbf{U}_{X, Y}$ is the joint distribution of $(X, Y)$ and $\mathbf{V}$ is the product of the marginal distributions of $X$ and $Y$. Formally, for every pair of $(x, y)$,

$$
\mathbf{U}_{X, Y}(X=x, Y=y)=\operatorname{Pr}[X=x, Y=y] \quad \mathbf{V}_{X, Y}(X=x, Y=y)=\operatorname{Pr}[X=x] \operatorname{Pr}[Y=y] .
$$

If $\mathbf{U}_{X, Y}$ is very different with $\mathbf{V}_{X, Y}$, then the mutual information between $X$ and $Y$ should be high, since knowing $X$ changes the belief for $Y$ a lot. If $\mathbf{U}_{X, Y}$ equals to $\mathrm{V}_{X, Y}$, then the mutual information between $X$ and $Y$ should be zero, since $X$ is independent with $Y$. Intuitively, the "distance" between $\mathbf{U}_{X, Y}$ and $\mathbf{V}_{X, Y}$ represents the mutual information between them.

Definition 4.1 ( $f$-mutual information). The $f$-mutual information between $X$ and $Y$ is defined as

$$
M I^{f}(X ; Y)=D_{f}\left(\mathbf{U}_{X, Y}, \mathbf{V}_{X, Y}\right)
$$

where $D_{f}$ is $f$-divergence.

Example $4.2($ KL Divergence and Shannon Mutual Information $I(\cdot ; \cdot))$. Choosing $f$-divergence as KL divergence, $f$-mutual information becomes the Shannon (conditional) mutual information (Cover and Thomas 2006)

$$
\begin{gathered}
I(X ; Y):=M I^{K L}(X ; Y)=\sum_{x, y} \operatorname{Pr}[X=x, Y=y] \log \frac{\operatorname{Pr}[X=x, Y=y]}{\operatorname{Pr}[X=x] \operatorname{Pr}[Y=y]}, \\
I(X ; Y \mid Z):=M I^{K L}(X ; Y \mid Z)=\sum_{x, y} \operatorname{Pr}[X=x, Y=y, Z=z] \log \frac{\operatorname{Pr}[X=x, Y=y \mid Z=z]}{\operatorname{Pr}[X=x \mid Z=z] \operatorname{Pr}[Y=y \mid Z=z]} .
\end{gathered}
$$

Example 4.3 (Total Variation Distance and TVD-mutual information MItvd $(\cdot ; \cdot)$ ). Choosing $f$ divergence as Total Variation Distance, $f$-mutual information becomes

$$
M^{t v d}(X ; Y):=\sum_{x, y}|\operatorname{Pr}[X=x, Y=y]-\operatorname{Pr}[X=x] \operatorname{Pr}[Y=y]| .
$$

For the strictness guarantee, we introduce the following definition:

Definition 4.4 (Fine-Grained Distribution). $P \in \Delta_{\Sigma_{X} \times \Sigma_{Y}}$ is a fine-grained joint distribution over $X$ and $Y$ if for every two distinct pairs $(x, y),\left(x^{\prime}, y^{\prime}\right), U_{X, Y}(X, Y):=P(X, Y)$ and $V_{X, Y}(X, Y):=$ $P(X) P(Y)$ can distinguish (see Definition 2.7) $(x, y)$ and $\left(x^{\prime}, y^{\prime}\right)$.

Theorem 4.5 (General Data-Processing Ineduality). When $f$ is (strictly) convex, $f$-mutual information $M I^{f}$ is information-monotone (and strictly information-monotone with respect to all finegrained joint distributions over $X$ and $Y)$. 
Definition 4.6 (Fine-Grained Prior). Given general setting $(n, \Sigma), Q$ is fine-grained prior if for every pair $i, j, Q\left(\Psi_{i}, \Psi_{j}\right)$ is a fine-grained joint distribution over $\Psi_{i}$ and $\Psi_{j}$.

Proof of Theorem 4.5. We will apply the information monotonicity of $f$-divergence to show the data-processing inequality of $f$-mutual information. We first introduce several matrix operations to ease the presentation of the proof.

Definition 4.7 (vec Operator (Henderson and Searle 1981)). The vec operator creates a column vector $\operatorname{vec}(A)$ from a matrix $A$ by stacking the column vectors of $A$.

Definition 4.8 (Kronecker Product (Henderson and Searle 1981)). The Kronecker product of two matrices $A \in \mathbb{R}^{m \times n}, B \in \mathbb{R}^{p \times q}$ is defined as the $m p \times n q$ matrix $A \otimes B=\left\{A_{i, j} B\right\}=\left[\begin{array}{ccc}A_{11} B & \ldots & A_{1 n} B \\ \vdots & \ddots & \vdots \\ A_{m 1} B & \ldots & A_{m n} B\end{array}\right]$.

Fact 4.9 (vec Operator and Kronecker Product (Roth 1934)). For any matrices $A \in \mathbb{R}^{n_{1} \times n_{2}}$, $X \in \mathbb{R}^{n_{2} \times n_{3}}, B \in \mathbb{R}^{n_{3} \times n_{4}}, \operatorname{vec}(A X B)=B^{T} \otimes A \operatorname{vec}(X)$.

Let $X: \Omega \mapsto \Sigma_{X}, Y: \Omega \mapsto \Sigma_{Y}$ be two random variables. $U_{X, Y}$ and $V_{X, Y}$ can be seen as two $\Sigma_{X} \times$ $\Sigma_{Y}$ matrices. Let $M$ be a $\left|\Sigma_{X}\right| \times\left|\Sigma_{X}\right|$ transition matrix.

We define $\Sigma_{X, Y}$ as $\Sigma_{X} \times \Sigma_{Y}$.

Note that the vectorization of the matrix that represents the probability measure over $X$ and $Y$ will not change the probability measure. Thus,

$$
D_{f}\left(U_{M(X), Y}, V_{M(X), Y}\right)=D_{f}\left(\operatorname{vec}\left(U_{M(X), Y}\right), \operatorname{vec}\left(V_{M(X), Y}\right)\right) .
$$

We define $I$ as a $\left|\Sigma_{Y}\right| \times\left|\Sigma_{Y}\right|$ identity matrix. For any transition matrix $M$, by simple calculations, we can see the Kronecker product between $M$ and the identity matrix $I$ is a transition matrix as well.

When $Y$ is independent with $M(X)$ conditioning on $X$, for any probability measure $P \in \Delta_{\Sigma_{X} \times \Sigma_{Y}}$ on $X$ and $Y$,

$$
\begin{aligned}
P\left(M(X)=x^{\prime}, Y=y\right) & =\sum_{x} P\left(M(X)=x^{\prime} \mid X=x, Y=y\right) P(X=x, Y=y) \\
& =\sum_{x} P\left(M(X)=x^{\prime} \mid X=x\right) P(X=x, Y=y)
\end{aligned}
$$

( $Y$ is independent with $M(X)$ conditioning on $X$ )

$$
\begin{aligned}
M I^{f}(M(X) ; Y) & =D_{f}\left(U_{M(X), Y}, V_{M(X), Y}\right) \\
& =D_{f}\left(\operatorname{vec}\left(U_{M(X), Y}\right), \operatorname{vec}\left(V_{M(X), Y}\right)\right) \\
& =D_{f}\left(\operatorname{vec}\left(M^{T} U_{X, Y} I\right), \operatorname{vec}\left(M^{T} V_{(X), Y} I\right)\right)
\end{aligned}
$$

(Equation (1), replacing $P$ by $U_{X, Y}$ and $V_{X, Y}$ )

$=D_{f}\left(I^{T} \otimes M^{T} \operatorname{vec}\left(U_{X, Y}\right), I^{T} \otimes M^{T} \operatorname{vec}\left(U_{X, Y}\right)\right)$

$\leq D_{f}\left(\operatorname{vec}\left(U_{X, Y}\right), \operatorname{vec}\left(V_{X, Y}\right)\right) \quad$ (information monotonicity of $f$-divergence)

$=D_{f}\left(U_{X, Y}, V_{X, Y}\right)$

$=M I^{f}(X ; Y)$.

Now we show the strictness guarantee. When $M$ is a non-permutation matrix, $\Theta:=\left(I^{T} \otimes\right.$ $\left.M^{T}\right)^{T}=M \otimes I$ is a non-permutation matrix as well. Thus there must exist $(x, y),\left(x^{\prime}, y^{\prime}\right),\left(x^{\prime \prime}, y^{\prime \prime}\right)$, such that both $\Theta\left((x, y),\left(x^{\prime}, y^{\prime}\right)\right)$ and $\Theta\left((x, y),\left(x^{\prime \prime}, y^{\prime \prime}\right)\right)$ are strictly positive where $\left(x^{\prime}, y^{\prime}\right) \neq$ $\left(x^{\prime \prime}, y^{\prime \prime}\right)$. According to the definition of fine-grained prior (see Definition 4.6), $U_{X, Y}$ and $V_{X, Y}$ can 
distinguish $\left(x^{\prime}, y^{\prime}\right)$ and $\left(x^{\prime}, y^{\prime}\right)$. Then Corollary 2.8 implies that the inequality in Equation (2) is strict.

Theorems 3.3 and 4.5 imply the following corollary.

Corollary 4.10. Given a general setting $(n, \Sigma)$, when $f$ is strictly convex and every agent's prior is fine-grained, the Mutual Information Paradigm MIP $\left(M I^{f}\right)$ is dominantly truthful and strongly truthful.

\subsection{Bregman Mutual Information}

It is natural to ask whether, in addition to $f$-divergence, we can use another commonly used divergence-Bregman divergence $D_{P S}$-to define an information-monotone information measure. Since the general Bregman divergence may not satisfy information monotonicity, the answer is likely to be negative. However, surprisingly, by properly using the Bregman divergence, we can obtain a new family of information measures $B M I^{P S}$ that satisfies almost all information-monotone properties of $f$-mutual information except for symmetry and one half of the data-processing inequality. Therefore, by plugging $B M I^{P S}$ into the Mutual Information Paradigm, we can still preserve the dominantly truthful property but lose the strongly truthful property, since an agent's lie cannot benefit herself but may benefit others.

Bregman Divergence (Bregman 1967; Gneiting and Raftery 2007). Bregman divergence $D_{P S}: \Delta_{\Sigma} \times$ $\Delta_{\Sigma} \rightarrow \mathbb{R}$ is a non-symmetric measure of the difference between distribution $\mathbf{p} \in \Delta_{\Sigma}$ and distribution $\mathrm{q} \in \Delta_{\Sigma}$ and is defined to be

$$
D_{P S}(\mathbf{p}, \mathbf{q})=P S(\mathbf{p}, \mathbf{p})-P S(\mathbf{p}, \mathbf{q}),
$$

where $P S$ is a proper scoring rule (see the definition of PS in Section 2.3).

Inspired by the $f$-mutual information, we can first try $D_{P S}\left(\mathbf{U}_{X, Y}, \mathbf{V}_{X, Y}\right)$ to define the Bregman mutual information. However, since the Bregman divergence may not satisfy the information monotonicity, this idea does not work. Intuitively, more information implies a more accurate prediction. Inspired by this intuition, we define Bregman mutual information between $X$ and $Y$ as an accuracy gain - the accuracy of the posterior $\operatorname{Pr}[\mathrm{Y} \mid X]$ minus the accuracy of the prior $\operatorname{Pr}[\mathrm{Y}]$. With this definition, if $X$ changes the belief for $Y$ a lot, then the Bregman mutual information between them is high; if $X$ is independent with $Y, \operatorname{Pr}[\mathrm{Y} \mid X]=\operatorname{Pr}[\mathrm{Y}]$, then the Bregman mutual information between them is zero.

We define $\mathbf{U}_{Y \mid X=x}$ and $\mathbf{U}_{Y}$ as two probability distribution over $Y$, such that

$$
\mathbf{U}_{Y \mid X=x}(Y=y)=\operatorname{Pr}[Y=y \mid X=x] \quad \mathbf{U}_{Y}(Y=y)=\operatorname{Pr}[Y=y] .
$$

Definition 4.11 (Bregman Mutual Information). The Bregman mutual information between $X$ and $Y$ is defined as

$$
B M I^{P S}(X ; Y)=\mathbb{E}_{X} D_{P S}\left(\mathrm{U}_{Y \mid X}, \mathrm{U}_{Y}\right)=\mathbb{E}_{X} P S(\operatorname{Pr}[\mathrm{Y} \mid X], \operatorname{Pr}[\mathrm{Y} \mid X])-P S(\operatorname{Pr}[\mathrm{Y} \mid X], \operatorname{Pr}[\mathrm{Y}]) .
$$

Bridging Log Scoring Rule and Shannon Mutual Information. Inspired by the definition of Bregman mutual information, we will show a novel connection between log scoring rule and Shannon information theory concepts-the log scoring rule can be used to construct an unbiased estimator of (conditional) Shannon mutual information. A powerful application of this connection is the information theoretic reconstruction of Prelec (2004) (Section 6.1.2).

The definition of Bregman mutual information says that the accuracy gain measured by a proper scoring rule $P S$ equals the information gain measured by the (conditional) Bregman mutual information $B M I^{P S}$. Theorem 4.12 shows that we can bridge the log scoring rule and Shannon mutual 
information by showing the accuracy gain measured by log scoring rule equals the information gain measured by (conditional) Shannon mutual information. Therefore, like $f$-mutual information, Bregman mutual information also generalizes Shannon mutual information (Corollary 4.13).

THEOREM 4.12 (EXPECTED ACCURACY GAIN = INFORMATION GAIN). For random variables $X, Y, Z$, when predicting $Y$, the logarithm score of prediction $\operatorname{Pr}[Y \mid Z, X]$ minus the logarithm score of prediction $\operatorname{Pr}[Y \mid Z]$ :

$$
\mathbb{E}_{X, Y, Z} L(Y, \operatorname{Pr}[Y \mid Z, X])-L(Y, \operatorname{Pr}[Y \mid Z])=I(X ; Y \mid Z),
$$

where $L: \sum \times \Delta_{\Sigma} \mapsto \mathbb{R}$ is the log scoring rule and $I(X ; Y \mid Z)$ is the Shannon mutual information between $X$ and $Y$ conditioning on $Z$.

Proof.

$$
\begin{aligned}
& \mathbb{E}_{X, Y, Z} L(Y, \operatorname{Pr}[Y \mid Z, X])-L(Y, \operatorname{Pr}[Y \mid Z]) \\
& \quad=\sum_{x, y, z} \operatorname{Pr}[X=x, Y=y, Z=z] \log \left(\frac{\operatorname{Pr}[Y=y \mid Z=z, X=x]}{\operatorname{Pr}[Y=y \mid Z=z]}\right) \\
& \quad=\sum_{x, y, z} \operatorname{Pr}[X=x, Y=y, Z=z] \log \left(\frac{\operatorname{Pr}[Y=y, X=x \mid Z=z]}{\operatorname{Pr}[Y=y \mid Z=z] \operatorname{Pr}[X=x \mid Z=z]}\right) \\
& =I(X ; Y \mid Z) .
\end{aligned}
$$

Recall that the conditional mutual information (Definition 3.2) is defined as

$$
\sum_{z} \operatorname{Pr}[Z=z] M I(X ; Y \mid Z=z)
$$

Thus,

$$
B M I^{P S}(X ; Y \mid Z)=\mathbb{E}_{X, Z} P S(\operatorname{Pr}[\mathrm{Y} \mid X, Z], \operatorname{Pr}[\mathrm{Y} \mid X, Z])-P S(\operatorname{Pr}[\mathrm{Y} \mid X, Z], \operatorname{Pr}[\mathrm{Y} \mid Z]),
$$

which is the accuracy of posterior $\operatorname{Pr}[\mathrm{Y} \mid X, Z]$ minus the accuracy of prior $\operatorname{Pr}[\mathrm{Y} \mid Z]$. Therefore, Fact 4.12 directly implies Corollary 4.13.

Corollary 4.13. BMI $I^{L(\cdot, \cdot)}(X ; Y \mid Z)=I(X ; Y \mid Z)$ where $B M I^{L(\cdot, \cdot)}$ is a Bregman mutual information that uses the log scoring rule $L(\cdot, \cdot)$ as the proper scoring rule.

Definition 4.14 (Quasi-Information-Monotone Mutual Information). We say MI is quasiinformation-monotone if and only if it is always non-negative and satisfies the data-processing inequality for the first entry.

We say $M I$ is strictly quasi-information-monotone with respect to a probability measure $P \in$ $\Delta_{\Sigma_{X} \times \Sigma_{Y}}$ if when the joint distribution over $X$ and $Y$ is $P$, for any non-permutation $M$, when $Y$ is independent with $M(X)$ conditioning on $X, M I(M(X) ; Y)<M I(X ; Y)$.

A quasi-information-monotone mutual information may not be symmetric. Thus, even if it satisfies the data-processing inequality for the first entry, it may not satisfy the data-processing inequality for the second entry, which means data-processing methods operating on $Y$ may increase $M I(X ; Y)$.

THEOREM 4.15. When PS is (strictly) proper, the Bregman mutual information is quasiinformation-monotone (and strictly quasi-information-monotone with respect to all fine-grained prior over $X$ and $Y$ ).

Intuitively, more information about $X$ provides a more accurate prediction for random variable $Y$. That is, $\operatorname{Pr}[\mathrm{Y} \mid M(X)]$ is less accurate than $\operatorname{Pr}[\mathrm{Y} \mid X]$. We will show the property of the proper 
scoring rules directly implies the above intuition and then the quasi-information-monotonicity of $B M I^{P S}$ follows.

Proof. The definition of proper scoring rules implies the non-negativity of Bregman divergence as well as that of Bregman mutual information.

For any transition probability $M$ that operates on $X$,

$$
\begin{aligned}
& B M I^{P S}(M(X) ; Y)= \mathbb{E}_{M(X)} P S(\operatorname{Pr}[\mathrm{Y} \mid M(X)], \operatorname{Pr}[\mathrm{Y} \mid M(X)])-P S(\operatorname{Pr}[\mathrm{Y} \mid M(X)], \operatorname{Pr}[\mathrm{Y}]) \\
&= \mathbb{E}_{M(X)} P S(\operatorname{Pr}[\mathrm{Y}], \operatorname{Pr}[\mathrm{Y} \mid M(X)])-P S(\operatorname{Pr}[\mathrm{Y}], \operatorname{Pr}[\mathrm{Y}]) \\
&\left(P S \text { is linear for the first entry and } \mathbb{E}_{M(X)} \operatorname{Pr}[\mathrm{Y} \mid M(X)]=\operatorname{Pr}[\mathrm{Y}]\right) \\
&= \mathbb{E}_{X, M(X)} P S(\operatorname{Pr}[\mathrm{Y} \mid X, M(X)], \operatorname{Pr}[\mathrm{Y} \mid M(X)])-P S(\operatorname{Pr}[\mathrm{Y}], \operatorname{Pr}[\mathrm{Y}]) \\
& \quad\left(P S \text { is linear for the first entry and } \operatorname{Pr}[\mathrm{Y}]=\mathbb{E}_{X, M(X)} \operatorname{Pr}[\mathrm{Y} \mid X, M(X)]\right) \\
&= \mathbb{E}_{X, M(X)} P S(\operatorname{Pr}[\mathrm{Y} \mid X], \operatorname{Pr}[\mathrm{Y} \mid M(X)])-P S(\operatorname{Pr}[\mathrm{Y}], \operatorname{Pr}[\mathrm{Y}]) \\
& \quad(\text { conditioning on } X, M(X) \text { is independent with } Y) \\
& \leq \mathbb{E}_{X} P S(\operatorname{Pr}[\mathrm{Y} \mid X], \operatorname{Pr}[\mathrm{Y} \mid X])-P S(\operatorname{Pr}[\mathrm{Y}], \operatorname{Pr}[\mathrm{Y}]) \\
&= \mathbb{E}_{X} P S(\operatorname{Pr}[\mathrm{Y} \mid X], \operatorname{Pr}[\mathrm{Y} \mid X])-P S(\operatorname{Pr}[\mathrm{Y} \mid X], \operatorname{Pr}[\mathrm{Y}]) \\
&\left(P S \text { is linear for the first entry and } \mathbb{E}_{X} \operatorname{Pr}[\mathrm{Y} \mid X]=\operatorname{Pr}[\mathrm{Y}]\right) \\
&= B M I^{P S}(X ; Y) .
\end{aligned}
$$

The inequality is strict when $P S$ is strictly proper and $\operatorname{Pr}[\mathrm{Y} \mid M(X)] \neq \operatorname{Pr}[\mathrm{Y} \mid X]$. When the prior over $X$ and $Y$ is fine-grained, $\operatorname{Pr}[\mathrm{Y} \mid M(X)] \neq \operatorname{Pr}[\mathrm{Y} \mid X]$ is valid if $M$ is a non-permutation transition. Thus, when PS is (strictly) proper, the Bregman mutual information is quasi-informationmonotone (and strictly quasi-information-monotone with respect to all fine-grained prior over $X$ and $Y)$.

THeOREM 4.16. Given a general setting $(n, \Sigma)$, when MI is strictly quasi-information-monotone with respect to every agent's prior, the Mutual Information Paradigm MIP(MI) is dominantly truthful.

For a quasi-information-monotone mutual information $M I$, data-processing methods operating on $Y$ may increase $M I(X ; Y)$ thus, the Mutual Information Paradigm MIP $(M I)$ may not be strongly truthful, since an agent's lie may benefit other agents.

Proof. The proof is the same as the dominantly truthfulness proof of Theorem 3.3. Note that proof only involves the data-processing inequality of the first entry of $M I$.

COROLLARY 4.17. Given a general setting $(n, \Sigma)$, when PS is strictly proper and every agent's prior is fine-grained, the Mutual Information Paradigm MIP $\left(B M I^{P S}\right)$ is dominantly truthful.

\section{MULTI-QUESTION, DETAIL FREE, MINIMAL SETTING}

In this section, we introduce the multi-question setting, which was previously studied in Dasgupta and Ghosh (2013) and Radanovic and Faltings (2014): $n$ agents are assigned the same $T$ questions (multi-questions). For each question $k$, each agent $i$ receives a private signal $\sigma_{i}^{k} \in \Sigma$ about question $k$ and is asked to report this signal. We call this setting $(n, T, \Sigma)$.

We see mechanisms in which agents are not required to report their forecasts for other agents' answer (minimal), and were the mechanism does not know the agents' priors (detail free). Agent $i$ may lie and report $\hat{\sigma}_{i}^{k} \neq \sigma_{i}^{k}$. Dasgupta and Ghosh (2013) give the following example for this setting: $n$ workers are asked to check the quality of $m$ goods, they may receive signal "high quality" or "low quality."

Agents have priors for questions. Each agent $i$ believes agents' private signals for question $k$ are chosen from a joint distribution $Q_{i}^{k}$ over $\Sigma^{n}$. Note that different agents may have different priors for the same question. 
In the multi-question setting, people usually make the following assumption:

Assumption 5.1 ( A PRIORI Similar AND RANDOM Order). For any $i$, any $k \neq k^{\prime}, Q_{i}^{k}=Q_{i}^{k^{\prime}}$. Moreover, all questions appear in a random order, independently drawn for each agent.

This means agents cannot distinguish each question without the private signal they receive.

We define $\left(\Psi_{1}, \Psi_{2}, \ldots, \Psi_{n}\right)$ as the joint random variables, such that

$$
\operatorname{Pr}\left(\Psi_{1}=\sigma_{1}, \Psi_{2}=\sigma_{2}, \ldots, \Psi_{n}=\sigma_{n}\right)
$$

equals the probability that agents $1,2, \ldots, n$ receive private signals $\left(\sigma_{1}, \sigma_{2}, \ldots, \sigma_{n}\right)$ correspondingly for a question that is picked uniformly at random.

We define $\left(\hat{\Psi}_{1}, \hat{\Psi}_{2}, \ldots, \hat{\Psi}_{n}\right)$ as the joint random variables, such that

$$
\operatorname{Pr}\left(\hat{\Psi}_{1}=\hat{\sigma}_{1}, \hat{\Psi}_{2}=\hat{\sigma}_{2}, \ldots, \hat{\Psi}_{n}=\hat{\sigma}_{n}\right)
$$

equals the probability that agents $1,2, \ldots, n$ reports signals $\left(\hat{\sigma}_{1}, \hat{\sigma}_{2}, \ldots, \hat{\sigma}_{n}\right)$ correspondingly a question that is picked uniformly at random. Note that the joint distribution over $\left(\hat{\Psi}_{1}, \hat{\Psi}_{2}, \ldots, \hat{\Psi}_{n}\right)$ depends on the strategies agents play.

For each question $k$, each agent $i$ 's strategy is $s_{i}^{k}$. We say agent $i$ plays a consistent strategy if for any $k, k^{\prime}, s_{i}^{k}=s_{i}^{k^{\prime}}$.

Recall that in the minimal mechanism, the strategy corresponds to a transition matrix. We define truth-telling $\mathrm{T}$ as the strategy where an agent truthfully reports her private signal for every question. T corresponds to the identity matrix. We say agent $i$ plays a permutation strategy if there exists a permutation transition matrix $\pi$, such that $s_{i}^{k}=\pi, \forall k$. Note that a permutation strategy is a consistent strategy. We define a consistent strategy profile as the strategy profile where all agents play a consistent strategy.

With the a priori similar and random order assumption, Dasgupta and Ghosh (2013) make the following observation:

ObServation 5.2 (DAsgupta AND GHosh 2013). When questions are a priori similar and agents receive questions in random order (Assumption 5.1), for every agent, using different strategies for different questions is the same as a mixed consistent strategy.

With the above observation, it is sufficient to only consider the consistent strategy profiles.

\subsection{The $f$-mutual Information Mechanism and Bregman Mutual Information Mechanism}

In this section, we give direct applications of the mutual information paradigm in the multiquestion setting-the $f$-mutual information mechanism and the Bregman mutual information mechanism. Both of them are a family of mechanisms that can be applied to the non-binary setting of multiple-choices questions and so generalize the results in Dasgupta and Ghosh (2013) that only applied to the binary setting. Moreover, both the $f$-mutual information mechanism and the Bregman mutual information mechanism are dominantly truthful. Later, we will map the mechanism in Dasgupta and Ghosh (2013) to a special case of the $f$-mutual information mechanism.

$f$-mutual Information Mechanism $\mathcal{M}_{M I^{f}}$. Given a multi-question setting $(n, T, \Sigma)$,

Report For each agent $i$, for each question $k$, she is asked to provide her private signal $\sigma_{i}^{k}$. We denote the actual answer she reports as $\hat{\sigma}_{i}^{k}$.

Payment/Information Score We arbitrarily pick a reference agent $j \neq i$. We define a probability measure $P$ over $\Sigma \times \Sigma$, such that $T * P\left(\hat{\Psi}_{i}=\sigma_{i} ; \hat{\Psi}_{j}=\sigma_{j}\right)$ equals the number of questions that agent $i$ answers $\sigma_{i}$ and agent $j$ answers $\sigma_{j}$. 
Agent $i$ is paid by her information score,

$$
M I^{f}\left(\hat{\Psi}_{i} ; \hat{\Psi}_{j}\right)
$$

where $\left(\hat{\Psi}_{i} ; \hat{\Psi}_{j}\right)$ draws from the probability measure $P$.

THEOREM 5.3. Given a multi-question setting $(n, T, \Sigma)$ with the a priori similar and random order Assumption (5.1), when the number of questions is infinite, $f$ is strictly convex and every agent's prior is fine-grained, the $f$-mutual information mechanism $\mathcal{M}_{M I f}$ is detail free, minimal, dominantly truthful, and strongly truthful.

Proof. We would like to show that the $f$-mutual information mechanism is the same as $\operatorname{MIP}\left(M I^{f}\right)$. Then Corollary 4.10 directly implies the theorem.

Based on Observation 5.2, it is sufficient to only consider consistent strategy profiles. When the number of questions is infinite and $\forall i$, agent $i$ plays the consistent strategy $s_{i}$,

$$
P\left(\hat{\Psi}_{i}=\sigma_{i} ; \hat{\Psi}_{j}=\sigma_{j}\right)=\operatorname{Pr}\left(\hat{\Psi}_{i}=\sigma_{i} ; \hat{\Psi}_{j}=\sigma_{j}\right)
$$

and $\hat{\Psi}_{i}=s_{i}\left(\Psi_{i}\right)$.

Therefore, with Assumption 5.1, when the number of questions is infinite, the $f$-mutual information mechanism is the same as $\operatorname{MIP}\left(M I^{f}\right)$ in the multi-question setting. Theorem 5.3 follows immediately from Corollary 4.10 .

Bregman Mutual Information Mechanism $\mathcal{M}_{B M I^{P S}}$. We can define the Bregman mutual information mechanism via the same definition of $f$-mutual information mechanism except replacing $M I^{f}$ by $B M I^{P S}$.

Corollary 4.17 directly implies the following theorem.

TheOREM 5.4. Given a multi-question setting $(n, T, \Sigma)$ with the a priori similar and random order Assumption (5.1), when the number of questions is infinite, PS is strictly proper and every agent's prior is fine-grained, the Bregman mutual information mechanism $\mathcal{M}_{B M I^{P S}}$ is detail free, minimal, and dominantly truthful.

Recall that since $B M I^{P S}$ only satisfies the data-processing inequality in one of the two coordinates, $\mathcal{M}_{B M I} P S$ is not strongly truthful in the sense that an agent's lie may benefit other agents.

\subsection{Mapping Dasgupta and Ghosh (2013), Correlated Agreement (CA) Mechanism (Shnayder et al. 2016) into Our Information Theoretic Framework}

This section maps Dasgupta and Ghosh (2013) to a special case of $f$-mutual information mechanism (in the binary setting) with the $f$-mutual information using the specific $f$-divergence, total variation distance. With the mapping, we can simplify the proof in Dasgupta and Ghosh (2013) to a direct application of our framework.

5.2.1 Prior Work. We first state the mechanism $M_{d}$ and the main theorem in Dasgupta and Ghosh (2013).

Mechanism $M_{d}$. Agents are asked to report binary signals 0 or 1 for each question. Uniformly randomly pick a reference agent $j$ for agent $i$. We denote $C_{i}$ as the set of questions agent $i$ answered. We denote $C_{j}$ as the set of questions agent $j$ answered. We denote $C_{i, j}$ as the set of questions both agent $i$ and agent $j$ answered. For each question $k \in C_{i, j}$ that both agent $i$ and agent $j$ answered, pick subsets $A \subseteq C_{i} \backslash k, B \subseteq C_{j} \backslash(k \cup A)$ with $|A|=|B|=d$. If such $A, B$ do not exist, then agent $i$ 's reward is 0 . Otherwise, we define $\overline{\hat{\sigma}}_{i}^{A}=\frac{\sum_{l \in A} \hat{\sigma}_{i}^{l}}{|A|}$ to be agent $i$ 's average answer for subset $A$, $\overline{\hat{\sigma}}_{j}^{B}=\frac{\sum_{l \in B} \hat{\sigma}_{j}^{l}}{|B|}$ is agent $j$ 's average answer for subset $B$. 
Agent $i$ 's reward for each question $k \in C_{i, j}$ is

$$
R_{i, j}^{k}:=\left[\hat{\sigma}_{i}^{k} * \hat{\sigma}_{j}^{k}+\left(1-\hat{\sigma}_{i}^{k}\right) *\left(1-\hat{\sigma}_{j}^{k}\right)\right]-\left[\overline{\hat{\sigma}}_{i}^{A} * \overline{\hat{\sigma}}_{j}^{B}+\left(1-\overline{\hat{\sigma}}_{i}^{A}\right) *\left(1-\overline{\hat{\sigma}}_{j}^{B}\right)\right]
$$

By simple calculations, essentially agent $i$ 's reward for each question $k \in C_{i, j}$ is the correlation between her answer and agent $j$ 's answer $-\mathbb{E}\left[\hat{\Psi}_{i} \hat{\Psi}_{j}\right]-\mathbb{E}\left[\hat{\Psi}_{i}\right] \mathbb{E}\left[\hat{\Psi}_{j}\right]$.

Assumption 5.5 (Informative). We assume agents' priors are informative in the sense that agents' private signals are strictly correlated/non-independent; i.e., for every $i, j$, the joint distribution over $\Psi_{i}, \Psi_{j}$ is not equal to the product of the marginal distributions over $\Psi_{i}, \Psi_{j}$.

Dasgupta and Ghosh (2013) also make an additional assumption:

AsSumption 5.6 (Positively CoRRELATED). Each question $k$ has a unknown ground truth $a^{k}$ and for every agent $i$, with probability greater or equal to $\frac{1}{2}$, agent $i$ receives private signal $a^{k}$.

We succinctly interpret the main results of Dasgupta and Ghosh (2013) as well as the results implied by the main results into the following theorem.

Theorem 5.7 (Dasgupta And Ghosh 2013). Given an multi-question setting $(n, T, \Sigma)$ with the a priori similar and random order Assumption (5.1), the positively correlated Assumption (5.6) and informative prior Assumption (5.5), when $T \geq d+1, M_{d}$ is informed truthful.

The parameter $d$ can be any positive integer. Larger $d$ will make the mechanism more robust. We will see $M_{d}$ equals a special case of the $f$-mutual information mechanism only if agent $i, j$ 's reported answers are positively correlated. Thus, $M_{d}$ is not dominantly truthful while the $f$-mutual information mechanism is. Although $M_{d}$ only requires a small number of questions, it only applies to binary choice questions, makes an extra assumption, and obtains weaker properties than the $f$-mutual information mechanism.

\subsubsection{Dasgupta and Ghosh (2013) $\approx$ TVD-mutual Information Mechanism.}

Proof Outline. We will first connect the expected payment in $M_{d}$ with a specific $f$-mutual information- $M I^{t v d}$. Then the result follows from the information monotone property of $f$-mutual information. Formally, we use the following claim to show the connection between mechanism $M_{d}$ and $f$-mutual information mechanism.

ClAIM $5.8\left(M_{d} \approx \mathcal{M}_{M I^{t v d}}\right)$. With a priori similar and random order assumption, in $M_{d}$, for every pairs $i, j$, for every reward question $k$,

$$
\mathbb{E}\left[R_{i, j}^{k}\right]=\frac{1}{2} M I^{t v d}\left(\Psi_{i} ; \Psi_{j}\right)
$$

if both of them play $\mathbf{T}$;

$$
\mathbb{E}\left[R_{i, j}^{k}\right] \leq \frac{1}{2} M I^{t v d}\left(\hat{\Psi}_{i} ; \hat{\Psi}_{j}\right)
$$

if one of them does not play $\mathrm{T}$.

Claim 5.8 shows the connection between $M_{d}$ and $\mathcal{M}_{M I^{t v d}}$. The only difference between $M_{d}$ and $\mathcal{M}_{M I^{t v d}}$ is that for agents $i, j$, when one of the agent does not play $\mathrm{T}$, the correlation between their reports is upper-bounded by rather than equal to the $t v d$-mutual information. Therefore, in $M_{d}$, truth-telling is not a dominant strategy. But the information-monotone property of $M I^{t v d}$ still guarantees the informed truthful property of $M_{d}$.

Proof of Theorem 5.7. We start to show the truthful property of $M_{d}$. 
For every agent $i$, given that everyone plays $\mathrm{T}$, when agent $i$ plays strategy $s_{i}$, agent $i$ 's expected payment for each reward question is

$$
\mathbb{E}\left[R_{i, j}^{k}\right] \leq \frac{1}{2} M I^{t v d}\left(\hat{\Psi}_{i} ; \Psi_{j}\right) \leq \frac{1}{2} M I^{t v d}\left(\Psi_{i} ; \Psi_{j}\right),
$$

since $M I^{t v d}$ is information-monotone. This implies the truthful property.

Moreover,

$$
\mathbb{E}\left[R_{i, j}^{k}\right] \leq \frac{1}{2} M I^{t v d}\left(\hat{\Psi}_{i} ; \hat{\Psi}_{j}\right) \leq \frac{1}{2} M I^{t v d}\left(\Psi_{i} ; \Psi_{j}\right) .
$$

Thus, the truth-telling strategy profile maximizes every agent's expected payment among all strategy profiles. When agents play uninformed strategies, their expected payments are less than zero, since the $f$-mutual information, including TVD-mutual information between two independent random variables, is zero. When agents are honest, their expected payments are strictly positive with the informative prior Assumption (5.5). This implies $M_{d}$ is informed truthful.

Proof for Claim 5.8. We first show that

$$
\mathbb{E}\left[R_{i, j}^{k}\right]=\frac{1}{2} M I^{t v d}\left(\Psi_{i} ; \Psi_{j}\right),
$$

if both of agents $i, j$ play $\mathbf{T}$.

Note that by simple calculations, Assumption 5.6 implies that for any $\sigma \in\{0,1\}$,

$$
\begin{gathered}
\operatorname{Pr}\left[\Psi_{j}=\sigma \mid \Psi_{i}=\sigma\right] \geq \operatorname{Pr}\left[\Psi_{j}=\sigma\right], \\
\operatorname{Pr}\left[\Psi_{j}=\sigma \mid \Psi_{i}=\sigma^{\prime}\right] \leq \operatorname{Pr}\left[\Psi_{j}=\sigma\right], \forall \sigma^{\prime} \neq \sigma .
\end{gathered}
$$

When both of agents $i, j$ play $\mathrm{T}$,

$$
\begin{aligned}
& \frac{1}{2} M I^{t v d}\left(\Psi_{i} ; \Psi_{j}\right)=\frac{1}{2} \sum_{\sigma, \sigma^{\prime}}\left|\operatorname{Pr}\left[\Psi_{i}=\sigma, \Psi_{j}=\sigma^{\prime}\right]-\operatorname{Pr}\left[\Psi_{i}=\sigma\right] \operatorname{Pr}\left[\Psi_{j}=\sigma^{\prime}\right]\right| \quad \text { (Definition of } M I^{t v d} \text { ) } \\
& =\frac{1}{2} \sum_{\sigma, \sigma^{\prime}} \mathbb{1}\left(\sigma=\sigma^{\prime}\right)\left(\operatorname{Pr}\left[\Psi_{i}=\sigma, \Psi_{j}=\sigma^{\prime}\right]-\operatorname{Pr}\left[\Psi_{i}=\sigma\right] \operatorname{Pr}\left[\Psi_{j}=\sigma^{\prime}\right]\right) \\
& +\mathbb{1}\left(\sigma \neq \sigma^{\prime}\right)\left(\operatorname{Pr}\left[\Psi_{i}=\sigma\right] \operatorname{Pr}\left[\Psi_{j}=\sigma^{\prime}\right]-\operatorname{Pr}\left[\Psi_{i}=\sigma, \Psi_{j}=\sigma^{\prime}\right]\right) \text { (Assumption 5.6) } \\
& =\sum_{\sigma}\left(\operatorname{Pr}\left[\Psi_{i}=\sigma, \Psi_{j}=\sigma\right]-\operatorname{Pr}\left[\Psi_{i}=\sigma\right] \operatorname{Pr}\left[\Psi_{j}=\sigma\right]\right) \\
& \text { Combining like terms, } \operatorname{Pr}[E]-\operatorname{Pr}[\neg E]=2 \operatorname{Pr}[E]-1) \\
& =\mathbb{E}\left[R_{i, j}^{k}\right] .
\end{aligned}
$$

The proof of

$$
\mathbb{E}\left[R_{i, j}^{k}\right] \leq \frac{1}{2} M I^{t v d}\left(\hat{\Psi}_{i} ; \hat{\Psi}_{j}\right)
$$

is similar to the above proof. We only need to replace $\Psi_{i}$ by $\hat{\Psi}_{i}$ and change the second equation to greater than, that is,

$$
\begin{aligned}
& \frac{1}{2} \sum_{\sigma, \sigma^{\prime}}\left|\operatorname{Pr}\left[\hat{\Psi}_{i}=\sigma, \hat{\Psi}_{j}=\sigma^{\prime}\right]-\operatorname{Pr}\left[\hat{\Psi}_{i}=\sigma\right] \operatorname{Pr}\left[\hat{\Psi}_{j}=\sigma^{\prime}\right]\right| \\
& \geq \frac{1}{2} \sum_{\sigma, \sigma^{\prime}} \mathbb{1}\left(\sigma=\sigma^{\prime}\right)\left(\operatorname{Pr}\left[\hat{\Psi}_{i}=\sigma, \hat{\Psi}_{j}=\sigma^{\prime}\right]-\operatorname{Pr}\left[\hat{\Psi}_{i}=\sigma\right] \operatorname{Pr}\left[\hat{\Psi}_{j}=\sigma^{\prime}\right]\right) \\
& \quad+\mathbb{1}\left(\sigma \neq \sigma^{\prime}\right)\left(\operatorname{Pr}\left[\hat{\Psi}_{i}=\sigma\right] \operatorname{Pr}\left[\hat{\Psi}_{j}=\sigma^{\prime}\right]-\operatorname{Pr}\left[\hat{\Psi}_{i}=\sigma, \hat{\Psi}_{j}=\sigma^{\prime}\right]\right) . \quad\left(\sum|x| \geq \sum x\right)
\end{aligned}
$$

We have finished the proof of Claim 5.8. 
5.2.3 Correlated Agreement (CA) Mechanism $\approx$ TVD-mutual Information Mechanism. The analysis in Section 5.2 is not restricted to Dasgupta and Ghosh (2013). We will show a non-binary extension of Dasgupta and Ghosh (2013), Shnayder et al. (2016)'s CA mechanism, is also a special case of $f$-mutual information mechanism-the TVD-mutual information mechanism $\mathcal{M}_{M I^{t v d}}$.

The correlation structure is defined as knowledge of the sign of all $\operatorname{Pr}\left[\Psi_{i}=\sigma, \Psi_{j}=\sigma^{\prime}\right]-\operatorname{Pr}\left[\Psi_{i}=\right.$ $\sigma] \operatorname{Pr}\left[\Psi_{j}=\sigma^{\prime}\right], \forall \sigma, \sigma^{\prime} \in \Sigma$. Note that the positively correlated setting is a special correlation structure where all signs are positive. CA applies to a more general setting than the positively correlated setting (Assumption 5.6) in the sense that CA assumes the knowledge of the correlation structure but the correlation structure does not need to be positively correlated. Here we give the analysis for CA in the special setting where the correlation structure is positively correlated. The analysis for other settings is similar.

The Correlated Agreement (CA) Mechanism (Shnayder et al. 2016). In the special setting where the correlation structure is positively correlated, the non-binary extension of $M_{d}$-the CA mechanism-can be reinterpreted as $M_{d}$ by replacing $R_{i, j}^{k}$ in $M_{d}$ by

$$
C_{i, j}^{k}:=\mathbb{1}\left(\hat{\sigma}_{i}^{k}=\hat{\sigma}_{j}^{k}\right)-\mathbb{1}\left(\hat{\sigma}_{i}^{\ell_{A}}=\hat{\sigma}_{j}^{\ell_{B}}\right),
$$

where $\ell_{A}$ is picked from subset $A$ uniformly at random and $\ell_{B}$ is picked from subset $B$ uniformly at random.

Theorem 5.9 (ShnAyder ET AL. 2016). Given an multi-question setting $(n, T, \Sigma)$ with the a priori similar and random order Assumption (5.1), informative prior Assumption (5.5) and the knowledge of the correlation structure, when $T \geq d+1$, the Correlated Agreement Mechanism is informed truthful.

Proof. In the special setting where the correlation structure is positively correlated, Claim 5.8 is still valid by replacing $R_{i, j}^{k}$ by $C_{i, j}^{k}$. The proof of Claim 5.8 that uses the definition of $R_{i, j}^{k}$,

$$
\begin{aligned}
\sum_{\sigma} & \left(\operatorname{Pr}\left[\Psi_{i}=\sigma, \Psi_{j}=\sigma\right]-\operatorname{Pr}\left[\Psi_{i}=\sigma\right] \operatorname{Pr}\left[\Psi_{j}=\sigma\right]\right) \\
& =\mathbb{E}\left[R_{i, j}^{k}\right],
\end{aligned}
$$

is still valid for $C_{i, j}^{k}$; i.e.,

$$
\begin{aligned}
\sum_{\sigma}\left(\operatorname{Pr}\left[\Psi_{i}=\sigma, \Psi_{j}=\sigma\right]-\operatorname{Pr}\left[\Psi_{i}=\sigma\right] \operatorname{Pr}\left[\Psi_{j}=\sigma\right]\right) \\
=\mathbb{E}\left[C_{i, j}^{k}\right] .
\end{aligned}
$$

Therefore, the proof of Theorem 5.9 is the same with the proof of Theorem 5.7. The analysis for other correlation structure settings is similar.

\section{SINGLE-QUESTION, DETAIL FREE, COMMON PRIOR, NON-MINIMAL SETTING}

In the single-question setting, agents are asked to answer a single question, which means that the mechanism can only obtain a single sample of all agents' private information $\left(\Psi_{1}, \Psi_{2}, \ldots, \Psi_{n}\right)$.

Assumption 6.1 (COMmon Prior). We assume agents have a common prior-it is a common knowledge that agents' private signals for the question are chosen from a common joint distribution $Q$ over $\Sigma^{n}$.

Assumption 6.2 (Symmetric Prior). We assume agents have a symmetric prior $Q-$ for any permutation $\pi:[n] \mapsto[n]$,

$$
Q\left(\Psi_{1}=\sigma_{\pi(1)}, \Psi_{2}=\sigma_{\pi(2)}, \ldots, \Psi_{n}=\sigma_{\pi(n)}\right)=Q\left(\Psi_{1}=\sigma_{1}, \Psi_{2}=\sigma_{2}, \ldots, \Psi_{n}=\sigma_{n}\right) .
$$


Because we will assume that the prior is symmetric, we denote the prior expectation for the fraction of agents who receives private signal $\sigma$ by $q(\sigma)$ and the posterior expectation for the fraction of agents who receives private signal $\sigma$, conditioning on one agent receiving signal $\sigma^{\prime}$ by $q\left(\sigma \mid \sigma^{\prime}\right)$. We also define $\mathbf{q}=q(\cdot)$ and $\mathbf{q}_{\sigma}=q(\cdot \mid \sigma)$.

With the above assumptions, agents who receive the same private signals will have the same prediction.

Assumption 6.3 (Informative Prior). We assume if agents have different private signals, they will have different expectations for the fraction of at least one signal. That is for any $\sigma \neq \sigma^{\prime}$, there exists $\sigma^{\prime \prime}$, such that $q\left(\sigma^{\prime \prime} \mid \sigma\right) \neq q\left(\sigma^{\prime \prime} \mid \sigma^{\prime}\right)$.

Note that with informative prior assumption, agents' private signals are strictly correlated. With the above assumptions, agents who receive different private signals will have different predictions.

\subsection{Mapping Bayesian Truth Serum into Our Information Theoretic Framework}

Bayesian Truth Serum (BTS) (Prelec 2004) rewards the agents whose answer is "surprisingly popular." In this section, we will show that in BTS, essentially each agent is paid the mutual information between her information and the aggregated information conditioning a random peer's information, which matches our Mutual Information Paradigm. We show this via the connection we found between the log scoring rule and Shannon mutual information-the accuracy gain equals the information gain. Mapping Bayesian Truth Serum into our information theoretic framework substantially simplifies the proof in Prelec (2004) via directly applying the information-monotone property of Shannon mutual information.

6.1.1 Prior Work. Prelec (2004) proposes the Bayesian Truth Serum mechanism in the singlequestion setting. In addition to the common prior and the symmetric prior assumptions, two additional assumptions are required:

Assumption 6.4 (Conditional Independence). We define the state of the world as a random variable $W: \Omega \mapsto \Delta_{\Sigma}$, such that given that $W=\omega$, agents' private signals are independently and identically distributed. That is, for every $i$, agent $i$ receives signal $\sigma$ with probability $\omega(\sigma)$.

Assumption 6.5 (LARge Group). The number of agents is infinite.

We define a random variable $\hat{W}: \Omega \mapsto \Delta_{\Sigma}$ such that its outcome is the distribution over agents' reported signals. The distribution over $\hat{W}$ dependes on all agents' strategies. With the large group assumption, when agents tell the truth, $\hat{W}=W$.

BTS uses $\hat{W}$ as the posterior distribution and uses agents' forecasts as the prior distribution, and then rewards agents for giving signal reports that are "unexpectedly common" with respect to this distribution. Intuitively, an agent will believe her private signal is underestimated by other agents, which means she will believe the actual fraction of her own private signal is higher than the average of agents' forecasts.

Prelec also proposes the signal-prediction framework for the design of detail free mechanisms in the single-question setting.

Signal-prediction Framework (Prelec 2004).Given a setting $(n, \Sigma)$ with a symmetric common prior $Q$, the signal-prediction framework defines a game in which each agent $i$ is asked to report his private signal $\sigma_{i} \in \Sigma$ and his prediction $\mathbf{p}_{i} \in \Delta_{\Sigma}$, a distribution over $\Sigma$, where $\mathbf{p}_{i}=\mathbf{q}_{\sigma_{i}}$. For any $\sigma \in \Sigma, \mathbf{p}_{i}(\sigma)$ is agent $i$ 's (reported) expectation for the fraction of other agents who has received $\sigma$ given he has received $\sigma_{i}$. However, agents may not tell the truth. In this framework, the report space $\mathcal{R}=\Sigma \times \Delta_{\Sigma}$. We define a report profile of agent $i$ as $r_{i}=\left(\hat{\sigma}_{i}, \hat{\mathbf{p}}_{i}\right) \in \mathcal{R}$ where $\hat{\sigma}_{i}$ is agent $i$ 's reported signal and $\hat{\mathbf{p}}_{i}$ is agent $i$ 's reported prediction. 
We would like to encourage truth-telling $\mathbf{T}$, namely that agent $i$ reports $\hat{\sigma}_{i}=\sigma_{i}, \hat{\mathbf{p}}_{i}=\mathbf{q}_{\sigma_{i}}$. To this end, agent $i$ will receive some payment $v_{i}\left(\hat{\sigma}_{i}, \hat{\mathbf{p}}_{i}, \hat{\sigma}_{-i}, \hat{\mathbf{p}}_{-i}\right)$ from the mechanism.

Mechanism Bayesian Truth Serum (BTS $(\alpha)$ ) (Prelec 2004). The Bayesian Truth Serum (BTS) follows the signal-prediction framework. Here, we introduce the payment of BTS. Each agent $i$ has two scores: a prediction score and an information score. BTS pays each agent

$$
\text { prediction score }+\alpha \cdot \text { information score, }
$$

where $\alpha>1$ To calculate the scores, for every agent $i$, the mechanism chooses a reference agent $j \neq i$ uniformly at random.

Agent $i$ 's information score is

$$
\operatorname{score}_{I m}\left(r_{i}, r_{j}\right):=\log \frac{f r\left(\hat{\sigma}_{i} \mid \hat{\sigma}_{-i}\right)}{\hat{\mathbf{p}}_{j}\left(\hat{\sigma}_{i}\right)}=\log f r\left(\hat{\sigma}_{i} \mid \hat{\boldsymbol{\sigma}}_{-i}\right)-\log \hat{\mathbf{p}}_{j}\left(\hat{\sigma}_{i}\right),
$$

where $\operatorname{fr}\left(\hat{\sigma}_{i} \mid \hat{\boldsymbol{\sigma}}_{-i}\right)$ is the fraction of all reported signals $\hat{\sigma}_{-i}$ (excluding agent $i$ ) that agree with agent $i$ 's reported signal $\hat{\sigma}_{i}$, which can be seen as the posterior expectation of the fraction of agents who report $\hat{\sigma}_{i}$ conditioning on all agents' reports, while $\hat{\mathbf{p}}_{j}\left(\hat{\sigma}_{i}\right)$ is agent $j$ 's posterior expectation of that fraction conditioning on agent $j$ 's private signal. Intuitively, the signals that actually occur more than other agents believe they will receive a higher information score.

Agent $i$ 's prediction score is

$$
\text { score }_{\text {Pre }}\left(r_{i}, r_{j}\right):=L\left(\hat{\sigma}_{j}, \hat{\mathbf{p}}_{i}\right)-\log f r\left(\hat{\sigma}_{j} \mid \hat{\boldsymbol{\sigma}}_{-j}\right)=\log \hat{\mathbf{p}}_{i}\left(\hat{\sigma}_{j}\right)-\log f r\left(\hat{\sigma}_{j} \mid \hat{\boldsymbol{\sigma}}_{-j}\right) .
$$

Note that only the log scoring rule part $L\left(\hat{\sigma}_{j}, \hat{\mathbf{p}}_{i}\right)$ is related to agent $i$ 's report. Based on the property of the log scoring rule, for agent $i$, to maximize her prediction score, the best $\hat{\mathbf{p}}_{i}(\sigma)$ should be her posterior expectation of the fraction of the agents who report $\sigma$ rather than receive.

Now we restate the main theorem concerning Bayesian Truth Serum:

TheOREM 6.6 (PRELeC 2004). With the common prior, the symmetric prior, the informative prior, the conditional independence, and the large group assumptions, BTS $(\alpha)$ is detail free, (i) truthful and (ii) the expected average information score when everyone tells the truth is higher than that in any other equilibrium, and strictly higher than that in any uninformed equilibrium. Moreover, (iii) for $\alpha>1$, BTS is informed truthful with respect to all equilibria.

Prelec (2004) uses some clever algebraic calculations to prove the main results. In the next section, we will apply our "accuracy gain=information gain" observation to map Bayesian Truth Serum (Prelec 2004) into our information theoretical framework and show results (ii) and (iii) via the data-processing inequality of Shannon mutual information. We put Prelec (2004)'s proof for results (i) in the Appendix, since it is already sufficiently simple and not very related to our framework.

6.1.2 Using Our Information Theoretic Framework to Analyze BTS. A key observation of BTS is that when agents report the optimal predictions, the average information score is exactly the "accuracy gain"-the accuracy of the posterior prediction for a random agent's report conditioning on all agents' reports, minus the accuracy of a random reference agent $j$ 's posterior prediction for the random agent's report conditioning on agent $j$ 's private signal. Based on Lemma 4.12, this accuracy gain equals the Shannon mutual information between a random agent's reported signal and all agents' reports conditioning on the random reference agent $j$ 's private signal $\Psi_{j}=\sigma_{j}$. Therefore, the expected information score can be represented as the form of Shannon mutual information. We have similar analysis for the prediction score. We formally state the above observation in Lemma 6.7. Aided by this lemma, we will show results (ii) and (iii) via applying the informationmonotone property of Shannon mutual information. 
LEMmA 6.7. In BTS, when agents tell the truth, each agent i's expected information score and prediction score are

$$
I\left(W ; \Psi_{i} \mid \Psi_{j}\right), \quad-I\left(W ; \Psi_{j} \mid \Psi_{i}\right),
$$

respectively, $\forall j \neq i$. When the agents play a non-truthful equilibrium, we denote random variable $\hat{\Psi}$ as the reported signal of an agent who is picked uniformly at random, the expected average information score and prediction score are

$$
I\left(\hat{W} ; \hat{\Psi} \mid \Psi_{j}\right), \quad-I\left(\hat{W} ; \hat{\Psi} \mid \Psi_{i}\right),
$$

respectively, $\forall i, j$.

Proof. When agents tell the truth, each agent $i$ 's expected information score is

$$
\begin{aligned}
\mathbb{E}_{\Psi_{i}, \Psi_{j}, W} L\left(\Psi_{i}, \operatorname{Pr}\left[\Psi_{i} \mid W\right]\right)-L\left(\Psi_{i}, \operatorname{Pr}\left[\Psi_{i} \mid \Psi_{j}\right]\right) & \\
& =\mathbb{E}_{\Psi_{i}, \Psi_{j}, W} L\left(\Psi_{i}, \operatorname{Pr}\left[\Psi_{i} \mid W, \Psi_{j}\right]\right)-L\left(\Psi_{i}, \operatorname{Pr}\left[\Psi_{i} \mid \Psi_{j}\right]\right) \\
& =I\left(W ; \Psi_{i} \mid \Psi_{j}\right),
\end{aligned}
$$

when she is paired with reference agent $j$. Since the prior is symmetric, $I\left(W ; \Psi_{i} \mid \Psi_{j}\right)$ is independent of the identity of $j$ if $j \neq i$.

In any equilibrium s, based on the properties of proper scoring rules, each agent $j$ will always maximize his expected prediction score by truthfully reporting his predictions. Moreover, for agent $j$, his reference agent is picked uniformly at random. Therefore,

$$
\hat{\mathbf{p}}_{j}(\hat{\sigma})=\operatorname{Pr}\left[\hat{\Psi}=\hat{\sigma} \mid \Psi_{j}=\sigma_{j}\right],
$$

where $\hat{\Psi}$ is the reported signal of an agent who is picked uniformly at random.

Note that when the number of agents is infinite, $\hat{W}$ contains all information we need to forecast a random person's report $\hat{\Psi}$; thus, we still have

$$
L(\hat{\Psi}, \operatorname{Pr}[\hat{\Psi} \mid \hat{W}])=L\left(\hat{\Psi}, \operatorname{Pr}\left[\hat{\Psi} \mid \hat{W}, \Psi_{j}\right]\right)
$$

Then we can replace $W, \Psi_{i}$ by $\hat{W}, \hat{\Psi}$ in the above equations and prove that the expected average information score is

$$
I\left(\hat{W} ; \hat{\Psi} \mid \Psi_{j}\right)
$$

The analysis for the expected prediction score is the same as the above analysis except that we need to exchange $i$ and $j$.

Proof of Theorem 6.6 (II), (III). Based on Lemma 6.7, when agents play an equilibrium, the expected average information score equals

$$
\begin{aligned}
I\left(\hat{W} ; \hat{\Psi} \mid \Psi_{j}\right) & =\sum_{\sigma_{j}} \operatorname{Pr}\left[\Psi_{j}=\sigma_{j}\right] I\left(\hat{W} ; \hat{\Psi} \mid \Psi_{j}=\sigma_{j}\right) \\
& \leq \sum_{\sigma_{j}} \operatorname{Pr}\left[\Psi_{j}=\sigma_{j}\right] I\left(\hat{W}, W ; \hat{\Psi} \mid \Psi_{j}=\sigma_{j}\right)
\end{aligned}
$$

(Data-processing inequality) 
Note that, when the number of agents is infinite, since every agent's strategy is independent with each other, we can see $W$ determines $\hat{W} .^{6}$ Therefore,

$$
\begin{aligned}
\sum_{\sigma_{j}} \operatorname{Pr} & {\left[\Psi_{j}=\sigma_{j}\right] I\left(\hat{W}, W ; \hat{\Psi} \mid \Psi_{j}=\sigma_{j}\right) } \\
& =\sum_{\sigma_{j}} \operatorname{Pr}\left[\Psi_{j}=\sigma_{j}\right] I\left(W ; \hat{\Psi} \mid \Psi_{j}=\sigma_{j}\right) \\
& \leq \sum_{\sigma_{j}} \operatorname{Pr}\left[\Psi_{j}=\sigma_{j}\right] I\left(W ; \Psi_{i} \mid \Psi_{j}=\sigma_{j}\right), \forall i \neq j
\end{aligned}
$$

(Data-processing inequality and the symmetric prior assumption)

$$
=I\left(W ; \Psi_{i} \mid \Psi_{j}\right), \forall i \neq j \text {. }
$$

Thus, the expected average information score is maximized when everyone tells the truth. Moreover, when agents play uninformed equilibrium, their expected average information score is zero, since the Shannon mutual information between two independent random variables is zero. When agents are honest, each agent's expected information score is strictly positive with the informative prior assumption.

It is left to show for $\alpha>1$, in $\operatorname{BTS}(\alpha)$, the agent-welfare is maximized by truth-telling over all equilibria. Lemma 6.7 shows that when the prior is symmetric, the sum of the expected prediction scores equals the sum of the expected information scores in any equilibrium. Thus, when $\alpha>1$, the agent welfare is proportional to the sum of the expected information scores. Then Property (iii) follows from Property (ii).

\section{CONCLUSION AND DISCUSSION}

We propose a simple yet powerful mechanism design framework, the Mutual Information Paradigm (MIP), for designing information elicitation mechanisms that reward truth-telling without verification. This framework shows a method to quantify participants' information without verification and pay them according to the amount of information they have.

A direct application of this framework produces two novel families of mechanisms, the $f$-mutual information mechanism and Bregman mutual information mechanism, which are the first mechanisms to achieve dominantly truthfulness, truth-telling maximizes the expected payment regardless of the other agents' strategies and for every agent, if she believes at least one other agent will tell the truth, truth-telling pays her strictly better than playing any "non-permutation" strategy., in the setting where agents do not need effort to receive their private information. We also map several mechanisms into our framework such that several existing and foundational results can be reconstructed easily.

For clean analysis, the current article is restricted to the setting where agents do not need to expend effort to receive their private information. In the setting where agents incur a cost for putting forth the effort required to obtain their private signal, the methods of this article still apply in a straightforward manner. However, the results obtained are slightly weaker. In particular, investing effort and truth-telling maximize the expected payment only when the agents believe at least a certain number of other agents invest effort and are honest. An earlier version (Kong and Schoenebeck 2016) of this article explicates this analysis.

\footnotetext{
${ }^{6}$ When $W=\omega, \hat{W}=\frac{1}{n} \sum_{i} M_{i}^{T} \omega$ where $M_{i}^{T}$ is the transpose matrix of the transition matrix corresponded to agent $i$ 's strategy for signal reporting, and the distribution $\omega$ is represented by a column vector.
} 
The $f$-mutual information mechanism (our mechanism for the multi-question setting) requires an infinite number of questions to calculate the $f$-mutual information. To apply the mechanism in practice, we can use the mutual information of the empirical distribution.

An important future direction is analyzing the sample complexity of calculating the $f$-mutual information and the influence of the choice of $f$. Our mutual information paradigm and $f$-mutual information mechanism apply to the setting where agents are heterogeneous as long as every agent only has a single private signal (i.e., no cheap signal exists). The current article does not apply to settings with "cheap signals" as discussed in Section 1.2. Additionally, the analysis in the single-question case does require that the prior is symmetric (the same across agents). Thus a future direction would be a better understanding of heterogeneous agents in the signal question setting.

\section{APPENDICES}

\section{A IMPOSSIBILITY (TIGHTNESS) RESULTS}

In this section, we will show an impossibility result that implies the optimality of the information theoretical framework. We will see when the mechanism knows no information about the prior profile, no non-trivial mechanism has truth-telling as the unique "best" equilibrium. Thus, it is too much to ask for a mechanism where truth-telling is paid strictly higher than any other nontruthful equilibrium. The best we can hope is to construct a mechanism where truth-telling is paid strictly higher than all non-truthful equilibria/strategy profiles excluding all permutation strategy profiles (Definition A.5) when the prior is symmetric; or all non-truthful equilibria/strategy profiles excluding all generalized permutation strategy profiles (Definition A.6) when the prior may be asymmetric. Because permutation strategies seem unnatural, risky, and contain the same amount of information with truth-telling.

Actually, we will show a much more general result in this section that is sufficiently strong to imply the optimality of the framework. Recall that a mechanism is strongly if truth-telling is strictly better than any other strategy profiles excluding generalized permutations strategy profiles. The results of this section imply that no truthful detail free mechanism can pay truth-telling $\mathrm{T}$ strictly better than all generalized permutations strategy profiles (Definition A.6) no matter what definition is the truth-telling strategy $\mathrm{T}$.

We omit the prior in the definition of strategy before, since we always fix the prior. However, when proving the impossibility results, the prior is not fixed. Therefore, we use the original definition of strategy in this section.

Definition A.1 (Strategy). Given a mechanism $\mathcal{M}$, we define the strategy of $\mathcal{M}$ for setting $(n, \Sigma)$ as a mapping $s$ from $(\sigma, Q)$ (private signal and prior) to a probability distribution over $\mathcal{R}$.

(Generalized) Permutation Strategy Profiles. A permutation $\pi: \Sigma \mapsto \Sigma$ can be seen as a relabelling of private information. Given two lists of permutations $\pi=\left(\pi_{1}, \pi_{2}, \ldots, \pi_{n}\right), \pi^{\prime}=\left(\pi_{1}^{\prime}, \pi_{2}^{\prime}, \ldots, \pi_{n}^{\prime}\right)$, we define the product of $\pi$ and $\pi^{\prime}$ as

$$
\pi \cdot \pi^{\prime}:=\left(\pi_{1} \cdot \pi_{1}^{\prime}, \pi_{2} \cdot \pi_{2}^{\prime}, \ldots, \pi_{n} \cdot \pi_{n}^{\prime}\right),
$$

where for every $i, \pi_{i} \cdot \pi_{i}^{\prime}$ is the group product of $\pi_{i}$ and $\pi_{i}^{\prime}$, such that $\pi_{i} \cdot \pi_{i}^{\prime}$ is a new permutation with $\pi_{i} \cdot \pi_{i}^{\prime}(\sigma)=\pi_{i}\left(\pi_{i}^{\prime}(\sigma)\right)$ for any $\sigma$.

We also define $\pi^{-1}$ as $\left(\pi_{1}^{-1}, \pi_{2}^{-1}, \ldots, \pi_{n}^{-1}\right)$.

By abusing notation a little, we define $\pi: Q \mapsto Q$ as a mapping from a prior $Q$ to a generalized permuted prior $\pi(Q)$ where for any $\sigma_{1}, \sigma_{2}, \ldots, \sigma_{n} \in \Sigma$,

$$
\pi(Q)\left(\sigma_{1}, \sigma_{2}, \ldots, \sigma_{n}\right)=Q\left(\pi_{1}^{-1}\left(\sigma_{1}\right), \pi_{2}^{-1}\left(\sigma_{2}\right), \ldots, \pi_{2}^{-1}\left(\sigma_{n}\right)\right),
$$


where $\sigma_{i}$ is the private signal of agent $i$. Notice that it follows that

$$
\pi(Q)\left(\pi_{1}\left(\sigma_{1}\right), \pi_{2}\left(\sigma_{2}\right), \ldots, \pi_{2}\left(\sigma_{n}\right)\right)=Q\left(\sigma_{1}, \sigma_{2}, \ldots, \sigma_{n}\right) .
$$

Intuitively, $\pi(Q)$ is the same as $Q$ after the signals are relabelled according to $\pi$.

Definition A.2 (Permutation List Operator on Strategy). For every agent $i$, given her strategy is $s_{i}$ and a permutation list $\pi$, we define $\pi\left(s_{i}\right)$ as the strategy, such that $\pi\left(s_{i}\right)(\sigma, Q)=s_{i}\left(\pi_{i}(\sigma), \pi(Q)\right)$ for every private information $\sigma$ and prior $Q$.

Definition A.3 (Permutation List Operator on Strategy Profile). Given a permutation list $\pi$, for any strategy profile $\mathbf{s}$, we define $\pi(\mathbf{s})$ as a strategy profile with $\pi(\mathbf{s})=\left(\pi\left(s_{1}\right), \pi\left(s_{2}\right), \ldots, \pi\left(s_{n}\right)\right)$.

Note that $\boldsymbol{\pi}^{-1} \boldsymbol{\pi} Q=Q$, which implies $\boldsymbol{\pi}^{-1} \boldsymbol{\pi}(\mathbf{s})=\mathbf{s}$.

We say $(\pi, \pi, \ldots, \pi)$ is a symmetric permutation list for any permutation $\pi$. For convenience, we write $(\pi, \pi, \ldots, \pi)(Q)$ as $\pi(Q),(\pi, \pi, \ldots, \pi)(s)$ as $\pi(s)$ and $(\pi, \pi, \ldots, \pi)(\boldsymbol{s})$ as $\pi(\boldsymbol{s})$.

We define a permutation strategy (profile) and then give a generalized version of this definition.

Definition A.4 (Permutation Strategy). We define a strategy $s$ as a permutation strategy if there exists a permutation $\pi$, such that $s=\pi(\mathrm{T})$.

Definition A.5 (Permutation Strategy Profile). We define a strategy profile $\mathbf{s}$ as a permutation strategy profile if there exists a permutation $\pi$, such that $s=\pi(T)$.

Definition A.6 (Generalized Permutation Strategy Profile). We define a strategy profile s as a generalized permutation strategy profile if there exists a permutation list $\pi=\left(\pi_{1}, \pi_{2}, \ldots, \pi_{n}\right)$, such that $\mathbf{s}=\boldsymbol{\pi}(\mathrm{T})=\left(\pi_{1}, \pi_{2}, \ldots, \pi_{n}\right)(\mathrm{T})$.

\section{A.1 Tightness Proof}

Definition A.7. Given a prior profile $\mathrm{Q}=\left(Q_{1}, Q_{2}, \ldots, Q_{n}\right)$ and a strategy profile $\mathbf{s}=$ $\left(s_{1}, s_{2}, \ldots, s_{n}\right)$, and a mechanism $\mathcal{M}$, for every agent $i$, we define

$$
v_{i}^{\mathcal{M}}(n, \Sigma, \mathbf{Q}, \mathbf{s})
$$

as agent $i$ 's ex ante expected payment when agents play $\mathbf{s}$ and all agents' private information is drawn from $Q_{i}$ that is, from agent $i$ 's viewpoint.

The impossibility result is stated as follows:

Proposition A.8. Let $\mathcal{M}$ be a mechanism that does not know the prior profile, then for any strategy profile s, and any permutation list $\pi$ :

(1) $\mathbf{s}$ is a strict Bayesian Nash equilibrium of $\mathcal{M}$ for any prior profile iff $\boldsymbol{\pi}(\mathbf{s})$ is a strict Bayesian Nash equilibrium of $\mathcal{M}$ for any prior profile.

(2) For every agent $i$, there exists a prior profile $\mathrm{Q}$, such that $v_{i}^{\mathcal{M}}(n, \Sigma, \mathrm{Q}, \mathbf{s}) \leq v_{i}^{\mathcal{M}}(n, \Sigma, \mathrm{Q}, \boldsymbol{\pi}(\mathbf{s}))$.

Additionally, if the mechanism knows the prior is symmetric, the above results only hold for any symmetric permutation list $(\pi, \pi, \ldots, \pi)$.

Proposition A.8 implies

Corollary A.9. Let $\mathcal{M}$ be a truthful mechanism, given truth-telling strategy $\mathrm{T}$,

when $\mathcal{M}$ knows no information about the prior profile of agents, if there exists a permutation list $\pi$, such that $\pi(\mathrm{T}) \neq \mathrm{T}, \mathrm{T}$ cannot be always paid strictly higher than all generalized permutation strategy profiles.

Additionally, if the mechanism knows the prior is symmetric, the above results only hold for any symmetric permutation list $(\pi, \pi, \ldots, \pi)$ and all permutation strategy profiles. 
We note that the requirement that there exists $\pi$, such that $\pi(T) \neq T$ only fails for very trivial mechanisms where the truthfully reported strategy does not depend on the signal an agent receives.

The key idea to prove this theorem is what we refer to as Indistinguishable Scenarios:

Definition A.10 (Scenario). We define a scenario for the setting $(n, \Sigma)$ as a tuple $(\mathrm{Q}, \mathbf{s})$ where $\mathbf{Q}$ is a prior profile, and $\mathbf{s}$ is a strategy profile.

Given mechanism $\mathcal{M}$, for any scenario $A=\left(Q_{A}, \mathbf{s}_{A}\right)$, we write $v_{i_{A}}^{\mathcal{M}}(n, \Sigma, A)$ as agent $i_{A}$ 's ex ante expected payment when agents play $\mathbf{s}_{A}$ and all agents' private signals are drawn from $Q_{i_{A}}$.

For two scenarios $A=\left(Q_{A}, \mathbf{s}_{A}\right), B=\left(Q_{B}, \mathbf{s}_{B}\right)$ for setting $(n, \Sigma)$, let $\sigma_{A}:=\left(\sigma_{1_{A}}, \sigma_{2_{A}}, \ldots, \sigma_{n_{A}}\right)$ be agents $\left(1_{A}, 2_{A}, \ldots, n_{A}\right)^{\prime}$ private signals, respectively, in scenario $A, \sigma_{B}:=\left(\sigma_{1_{B}}, \sigma_{2_{B}}, \ldots, \sigma_{n_{B}}\right)$ be agents $\left(1_{B}, 2_{B}, \ldots, n_{B}\right)^{\prime}$ 'private signals, respectively, in scenario $B$.

Definition A.11 (Indistinguishable Scenarios). We say two scenarios $A, B$ are indistinguishable $A \approx B$ if there is a coupling of the random variables $\sigma_{A}$ and $\sigma_{B}$, such that $\forall i, s_{i_{A}}\left(\sigma_{i_{A}}, Q_{i_{A}}\right)=$ $s_{i_{B}}\left(\sigma_{i_{B}}, Q_{i_{B}}\right)$ and agent $i_{A}$ has the same belief about the world as agent $i_{B}$, in other words, for every $j, \operatorname{Pr}\left(\hat{r}_{j_{A}}=\hat{r} \mid \sigma_{i_{A}}, \mathrm{Q}_{A}, \mathbf{s}_{A}\right)=\operatorname{Pr}\left(\hat{r}_{j_{B}}=\hat{r} \mid \sigma_{i_{B}}, \mathrm{Q}_{B}, \mathbf{s}_{B}\right) \forall \hat{r} \in \mathcal{R}$.

Now we will prove two properties of indistinguishable scenarios, which are the main tools in the proof for our impossibility result.

Observation A.12. If $\left(\mathbf{Q}_{A}, \mathbf{s}_{A}\right) \approx\left(\mathrm{Q}_{B}, \mathbf{s}_{B}\right)$, then (i) for any mechanism $\mathcal{M}, \mathbf{s}_{A}$ is a (strict) equilibrium for the prior profile $Q_{A}$ iff $\mathbf{s}_{B}$ is a (strict) equilibrium for the prior profile $Q_{B}$. (ii) $\forall i$, $v_{i_{A}}^{\mathcal{M}}(n, \Sigma, A)=v_{i_{B}}^{\mathcal{M}}(n, \Sigma, B)$

At a high level, (1) is true, since any reported profile distribution that agent $i_{A}$ can deviate to, agent $i_{B}$ can deviate to the same reported profile distribution as well and obtain the same expected payment as agent $i_{A}$.

Formally, we will prove the $\Rightarrow$ direction in (1) by contradiction. The proof of the other direction will be similar. Consider the coupling for $\sigma_{A}, \sigma_{B}$ mentioned in the definition of indistinguishable scenarios. For the sake of contradiction, assume there exists $i$ and $\sigma_{i_{B}}$, such that $\hat{r}^{\prime} \neq s_{i_{B}}\left(\sigma_{i_{B}}, Q_{i_{A}}\right)$ is a best response for agent $i_{B}$. Since agent $i_{A}$ has the same belief about the world as agent $i_{B}$ and $s_{i_{A}}\left(\sigma_{i_{A}}, Q_{i_{A}}\right)=s_{i_{B}}\left(\sigma_{i_{B}}, Q_{i_{B}}\right), \hat{r}^{\prime} \neq s_{i_{A}}\left(\sigma_{i_{A}}, Q_{i_{A}}\right)$ is a best response to agent $i_{A}$ as well, which is a contradiction to the fact that $\mathbf{s}_{A}$ is a strictly equilibrium for prior $Q_{i_{A}}$.

To gain intuition about (2), consider the coupling again. For any $i$, agent $i_{A}$ reports the same thing and has the same belief for the world as agent $i_{B}$, which implies the expected payoff of agent $i_{A}$ is the same as agent $i_{B}$. (2) follows.

Now we are ready to prove our impossibility result:

Proof of Proposition A.8. We prove part (1) and part (2) separately.

Proof of Part (1). Let $A:=(\mathrm{Q}, \mathrm{s}), B:=\left(\pi^{-1}(\mathrm{Q}), \boldsymbol{\pi}(\mathrm{s})\right)$. We will show that for any strategy profile s and any prior $Q, A \approx B$. Based on our above observations, part (1) immediately follows from that fact.

To prove $(Q, \mathbf{s}) \approx\left(\boldsymbol{\pi}^{-1} Q, \boldsymbol{\pi}(\mathbf{s})\right)$, for every $i$, we can couple $\left(\sigma_{1}, \sigma_{2}, \ldots, \sigma_{n}\right)$ with

$\left(\pi_{1}^{-1}\left(\sigma_{1}\right), \pi_{2}^{-1}\left(\sigma_{2}\right), \ldots, \pi_{n}^{-1}\left(\sigma_{n}\right)\right)$ where $\left(\sigma_{1}, \sigma_{2}, \ldots, \sigma_{n}\right)$ is drawn from $Q_{i}$. It is a legal coupling, since

$$
\pi^{-1}\left(Q_{i}\right)\left(\pi_{1}^{-1}\left(\sigma_{1}\right), \pi_{2}^{-1}\left(\sigma_{2}\right), \ldots, \pi_{n}^{-1}\left(\sigma_{n}\right)\right)=Q_{i}\left(\sigma_{1}, \sigma_{2}, \ldots, \sigma_{n}\right),
$$

according to the definition of $\pi^{-1}(Q)$.

Now we show this coupling satisfies the condition in Definition A.11. First note that 


$$
\begin{aligned}
\pi\left(s_{i}\right)\left(\pi^{-1}\left(\sigma_{i}\right), \pi^{-1}(Q)\right)=s_{i} & \left(\sigma_{i}, Q\right) . \text { Now we begin to calculate } \operatorname{Pr}\left(\hat{r}_{j_{B}}=\hat{r} \mid \sigma_{i_{B}}, \mathbf{Q}_{B}, \mathbf{s}_{B}\right) \\
\operatorname{Pr}\left(\hat{r}_{j_{B}}=\hat{r} \mid \sigma_{i_{B}}, Q_{B}, \mathbf{s}_{B}\right) & =\operatorname{Pr}\left(\hat{r}_{j_{B}}=\hat{r} \mid \pi_{i}^{-1}\left(\sigma_{i_{A}}\right), \pi^{-1}\left(Q_{j_{A}}\right), \pi\left(\mathbf{s}_{A}\right)\right) \\
& =\sum_{\sigma^{\prime}} \pi^{-1}\left(Q_{i_{A}}\right)\left(\sigma^{\prime} \mid \pi_{i}^{-1}\left(\sigma_{i_{A}}\right)\right) \operatorname{Pr}\left(\pi\left(s_{j_{A}}\right)\left(\sigma^{\prime}, \boldsymbol{\pi}^{-1}\left(Q_{j_{A}}\right)\right)=\hat{r}\right) \\
& =\sum_{\sigma^{\prime}} \pi^{-1}\left(Q_{i_{A}}\right)\left(\sigma^{\prime} \mid \pi_{i}^{-1}\left(\sigma_{i_{A}}\right)\right) \operatorname{Pr}\left(s_{j_{A}}\left(\pi\left(\sigma^{\prime}\right), \pi \pi^{-1}\left(Q_{j_{A}}\right)\right)=\hat{r}\right) \\
& =\sum_{\sigma^{\prime}} Q_{i_{A}}\left(\pi_{j}\left(\sigma^{\prime}\right) \mid \sigma_{i_{A}}\right) \operatorname{Pr}\left(s_{j_{A}}\left(\pi\left(\sigma^{\prime}\right), Q_{j_{A}}\right)=\hat{r}\right) \\
& =\sum_{\sigma^{\prime \prime}} Q_{i_{A}}\left(\sigma^{\prime \prime} \mid \sigma_{i_{A}}\right) \operatorname{Pr}\left(s_{j_{A}}\left(\sigma^{\prime \prime}, Q_{i_{A}}\right)=\hat{r}\right) \\
& =\operatorname{Pr}\left(\hat{r}_{j_{A}}=\hat{r} \mid \sigma_{i_{A}}, Q_{A}, s_{A}\right) .
\end{aligned}
$$

From Equations (3) and (4): To calculate the probability that agent $j_{B}$ has reported $\hat{r}$, we should sum over all possible private signals agent $j_{B}$ has received and calculate the probability agent $j_{B}$ reported $\hat{r}$ conditioning on he received private signal $\sigma^{\prime}$, which is determined by agent $j_{B}$ 's strategy $\pi\left(s_{j_{A}}\right)$.

By abusing notation a little bit, we can write $\pi\left(s_{j_{A}}\right)\left(\sigma^{\prime}, \pi^{-1} Q_{j_{A}}\right)$ as a random variable (it is actually a distribution) with $\operatorname{Pr}\left(\boldsymbol{\pi}\left(s_{j_{A}}\right)\left(\sigma^{\prime}, \boldsymbol{\pi}^{-1} Q\right)=\hat{r}\right)=\pi\left(s_{j_{A}}\right)\left(\sigma^{\prime}, \boldsymbol{\pi}^{-1} Q\right)(\hat{r})$. According to above explanation, Equation (4) follows.

Equation (5) follows from the definition of permuted strategy.

Equation (6) follows from the definition of permuted prior.

By replacing $\pi_{j}\left(\sigma^{\prime}\right)$ by $\sigma^{\prime \prime}$, Equation (7) follows.

We finished the proof $A \approx B$, as previously argued, result (1) follows.

Proof for Part (2). We will prove the second part by contradiction:

Fix permutation strategy profile $\pi$. First notice that there exists a positive integer $O_{d}$, such that $\pi^{O_{d}}=I$, where $I$ is the identity and agents play $I$ means they tell the truth.

Given any strategy profile $s$, for the sake of contradiction, we assume that there exists a mechanism $\mathcal{M}$ with unknown prior profile, such that $v_{i_{A}}^{\mathcal{M}}(n, \Sigma, \mathbf{Q}, \mathbf{s})>v_{i_{A}}^{\mathcal{M}}(n, \Sigma, \mathbf{Q}, \pi(\mathbf{s}))$ for any prior $Q$. For positive integer $k \in\left\{0,1, \ldots, O_{d}\right\}$, we construct three scenarios:

$$
A_{k}:=\left(\pi^{k}(\mathrm{Q}), \mathbf{s}\right), A_{k+1}:=\left(\pi^{k+1}(\mathrm{Q}), \mathbf{s}\right), B_{k}:=\left(\pi^{k}(\mathrm{Q}), \pi(\mathrm{s})\right)
$$

and show for any $k$,

(I) $v_{i_{A}}^{\mathcal{M}}\left(n, \Sigma, A_{k}\right)>v_{i_{A}}^{\mathcal{M}}\left(n, \Sigma, B_{k}\right)$,

(II) $v_{i_{A}}^{\mathcal{M}}\left(n, \Sigma, A_{k+1}\right)=v_{i_{A}}^{\mathcal{M}}\left(n, \Sigma, B_{k}\right)$.

Combining (I), (II), and the fact $A_{0}=A_{O_{d}}$, we have

$$
v_{i_{A}}^{\mathcal{M}}\left(n, \Sigma, A_{0}\right)>v_{i_{A}}^{\mathcal{M}}\left(n, \Sigma, A_{1}\right)>\cdots v_{i_{A}}^{\mathcal{M}}\left(n, \Sigma, A_{O_{d}}\right)=v_{i_{A}}^{\mathcal{M}}\left(n, \Sigma, A_{0}\right),
$$

which is a contradiction.

Now it is only left to show (I) and (II). Based on our assumption,

$$
v_{i_{A}}^{\mathcal{M}}(n, \Sigma, Q, s)>v_{i_{A}}^{\mathcal{M}}(n, \Sigma, Q, \pi(s)),
$$

for any prior $Q$, we have (I). By the same proof we have in part (1), we have $A_{k+1} \approx B_{k}$, which implies (II) according to our above observations.

When the mechanism knows the prior is symmetric, the above proof is still valid if we replace the permutation list $\pi$ by symmetric permutation list $(\pi, \pi, \ldots, \pi)$. 


\section{B PROOF OF THE TRUTHFULNESS OF BTS}

Proof of Theorem 6.6 (I) (Prelec 2004). When everyone else tells the truth, for every $i$, agent $i$ will report truthful $\mathbf{p}_{i}$ to maximize her expected prediction score based on the properties of log scoring rule. Thus, $\hat{\mathbf{p}}_{i}=\mathrm{p}_{i}$ for every $i$.

For the expected information score, we want to calculate the optimal $\sigma$ agent $i$ should report to maximize her expected information score when everyone else tells the truth, given that she receives $\Psi_{i}=\sigma_{i}$.

$$
\begin{aligned}
& \arg _{\max } \mathbb{E}_{\Psi_{j}, W \mid \Psi_{i}=\sigma_{i}} \log \left(\frac{\operatorname{Pr}\left[\Psi_{i}=\sigma \mid W\right]}{\operatorname{Pr}\left[\Psi_{i}=\sigma \mid \Psi_{j}\right]}\right) \\
& =\arg _{\max _{\sigma}} \mathbb{E}_{\Psi_{j}, W \mid \Psi_{i}=\sigma_{i}} \log \left(\frac{\operatorname{Pr}\left[\Psi_{i}=\sigma \mid W, \Psi_{j}\right]}{\operatorname{Pr}\left[\Psi_{i}=\sigma \mid \Psi_{j}\right]}\right) \quad \text { (Conditional independence) } \\
& =\arg _{\max _{\sigma}} \mathbb{E}_{\Psi_{j}, W \mid \Psi_{i}=\sigma_{i}} \log \left(\frac{\operatorname{Pr}\left[\Psi_{i}=\sigma, W \mid \Psi_{j}\right]}{\operatorname{Pr}\left[\Psi_{i}=\sigma \mid \Psi_{j}\right] \operatorname{Pr}\left[W \mid \Psi_{j}\right]}\right) \\
& =\arg _{\max _{\sigma}} \mathbb{E}_{\Psi_{j}, W \mid \Psi_{i}=\sigma_{i}} \log \left(\frac{\operatorname{Pr}\left[W \mid \Psi_{i}=\sigma, \Psi_{j}\right]}{\operatorname{Pr}\left[W \mid \Psi_{j}\right]}\right) \\
& =\arg _{\max _{\sigma}} \mathbb{E}_{\Psi_{j}} L\left(\operatorname{Pr}\left[W \mid \Psi_{i}=\sigma_{i}, \Psi_{j}\right], \operatorname{Pr}\left[W \mid \Psi_{i}=\sigma, \Psi_{j}\right]\right) \\
& \text { (we can add } \log \operatorname{Pr}\left[W \mid \Psi_{j}\right] \text {, which is independent of } \sigma \text { ) } \\
& =\sigma_{i} . \\
& \left(\arg \max _{\mathbf{q}} L(\mathbf{p}, \mathbf{q})=\mathbf{p}\right)
\end{aligned}
$$

Therefore, in BTS, for every $i$, agent $i$ 's best response is $\left(\sigma_{i}, \mathbf{p}_{i}\right)$ when everyone else tells the truth. BTS is truthful.

\section{PROOF OF INFORMATION MONOTONICITY OF F-DIVERGENCE}

Fact C.1 (Information Monotonicity ((Ali And Silvey 1966; Amari ANd Cichocki 2010; Liese AND VAJDA 2006))). For any strictly convex function $f$, $f$-divergence $D_{f}(\mathbf{p}, \mathbf{q})$ satisfies information monotonicity so that for any transition matrix $\theta \in \mathbb{R}^{|\Sigma| \times|\Sigma|}, D_{f}(\mathbf{p}, \mathbf{q}) \geq D_{f}\left(\theta^{T} \mathbf{p}, \theta^{T} \mathbf{q}\right)$.

Moreover, the inequality is strict if and only if there exists $\sigma, \sigma^{\prime}, \sigma^{\prime \prime}$, such that $\frac{\mathbf{p}\left(\sigma^{\prime \prime}\right)}{\mathbf{p}\left(\sigma^{\prime}\right)} \neq \frac{\mathbf{q}\left(\sigma^{\prime \prime}\right)}{\mathbf{q}\left(\sigma^{\prime}\right)}$ and $\theta_{\sigma^{\prime}, \sigma} \mathbf{p}\left(\sigma^{\prime}\right)>0, \theta_{\sigma^{\prime \prime}, \sigma} \mathbf{p}\left(\sigma^{\prime \prime}\right)>0$.

If the strictness condition does not satisfied, then we can see $\theta^{T} \mathbf{p}$ and $\theta^{T} \mathbf{q}$ are $\mathbf{p}$ and q's sufficient statistic, which means the transition $\theta$ does not lose any information, thus, the equality holds.

Proof. The proof follows from algebraic manipulation and one application of convexity.

$$
\begin{aligned}
& D_{f}\left(\theta^{T} \mathbf{p}, \theta^{T} \mathbf{q}\right)=\sum_{\sigma}\left(\theta^{T} \mathbf{p}\right)(\sigma) f\left(\frac{\left(\theta^{T} \mathbf{q}\right)(\sigma)}{\left(\theta^{T} \mathbf{p}\right)(\sigma)}\right) \\
& =\sum_{\sigma} \theta_{\sigma,}^{T} \mathbf{p} f\left(\frac{\theta_{\sigma, \cdot \mathbf{q}}^{T}}{\theta_{\sigma, \cdot \mathbf{p}}^{T}}\right) \\
& =\sum_{\sigma} \theta_{\sigma,}^{T} \mathbf{p} f\left(\frac{1}{\theta_{\sigma, \cdot \mathbf{p}}^{T}} \sum_{\sigma^{\prime}} \theta_{\sigma, \sigma^{\prime}}^{T} \mathbf{p}\left(\sigma^{\prime}\right) \frac{\mathbf{q}\left(\sigma^{\prime}\right)}{\mathbf{p}\left(\sigma^{\prime}\right)}\right) \\
& \leq \sum_{\sigma} \theta_{\sigma, \cdot}^{T} \mathbf{p} \frac{1}{\theta_{\sigma, \cdot \mathbf{p}}^{T}} \sum_{\sigma^{\prime}} \theta_{\sigma, \sigma^{\prime}}^{T} \mathbf{p}\left(\sigma^{\prime}\right) f\left(\frac{\mathbf{q}\left(\sigma^{\prime}\right)}{\mathbf{p}\left(\sigma^{\prime}\right)}\right) \\
& =\sum_{\sigma} \mathbf{p}(\sigma) f\left(\frac{\mathbf{q}(\sigma)}{\mathbf{p}(\sigma)}\right)=D_{f}(\mathbf{p}, \mathbf{q}) \text {. }
\end{aligned}
$$


The second equality holds, since $\left(\theta^{T} \mathbf{p}\right)(\sigma)$ is dot product of the $\sigma^{\text {th }}$ row of $\theta^{T}$ and $\mathbf{p}$.

The third equality holds, since $\sum_{\sigma^{\prime}} \theta_{\sigma, \sigma^{\prime}}^{T} \mathbf{p}\left(\sigma^{\prime}\right) \frac{\mathbf{q}\left(\sigma^{\prime}\right)}{\mathbf{p}\left(\sigma^{\prime}\right)}=\theta_{\sigma, \cdot \mathbf{q}}^{T}$.

The fourth inequality follows from the convexity of $f(\cdot)$.

The last equality holds, since $\sum_{\sigma} \theta_{\sigma, \sigma^{\prime}}^{T}=1$.

We now examine under what conditions the inequality in Equation (12) is strict. Note that for any strictly convex function $g$, if $\forall u, \lambda_{u}>0$, then $g\left(\sum_{u} \lambda_{u} x_{u}\right)=\sum_{u} \lambda_{u} g\left(x_{u}\right)$ if and only if there exists $x$, such that $\forall u, x_{u}=x$. By this property, the inequality is strict if and only if there exists $\sigma, \sigma^{\prime}, \sigma^{\prime \prime}$, such that $\frac{\mathrm{q}\left(\sigma^{\prime}\right)}{\mathbf{p}\left(\sigma^{\prime}\right)} \neq \frac{\mathrm{q}\left(\sigma^{\prime \prime}\right)}{\mathbf{p}\left(\sigma^{\prime \prime}\right)}$ and $\theta_{\sigma, \sigma^{\prime}}^{T} \mathbf{p}\left(\sigma^{\prime}\right)>0, \theta_{\sigma, \sigma^{\prime \prime}}^{T} \mathbf{p}\left(\sigma^{\prime \prime}\right)>0$.

\section{ACKNOWLEDGMENT}

The authors thank David Parkes for providing helpful suggestions.

\section{REFERENCES}

Arpit Agarwal, Debmalya Mandal, David C. Parkes, and Nisarg Shah. 2017. Peer prediction with heterogeneous users. In Proceedings of the ACM Conference on Economics and Computation (EC'17). 81-98.

Syed Mumtaz Ali and Samuel D. Silvey. 1966. A general class of coefficients of divergence of one distribution from another. f. Roy. Stat. Soc. Ser. B (Methodol.) 28, 1 (1966), 131-142.

S.-I. Amari and A. Cichocki. 2010. Information geometry of divergence functions. Bull. Polish Acad. Sci.: Tech. Sci. 58, 1 (2010), 183-195.

Lev M. Bregman. 1967. The relaxation method of finding the common point of convex sets and its application to the solution of problems in convex programming. USSR Comput. Math. Math. Phys. 7, 3 (1967), 200-217.

Thomas M. Cover and Joy A. Thomas. 2006. Elements of Information Theory, 2nd ed. Wiley Interscience.

Imre Csiszár, Paul C. Shields et al. 2004. Information theory and statistics: A tutorial. Found. Trends Commun. Info. Theory 1,4 (2004), 417-528.

Anirban Dasgupta and Arpita Ghosh. 2013. Crowdsourced judgement elicitation with endogenous proficiency. In Proceedings of the 22nd International Conference on World Wide Web. International World Wide Web Conferences Steering Committee, 319-330.

Boi Faltings, Radu Jurca, Pearl Pu, and Bao Duy Tran. 2014. Incentives to counter bias in human computation. In Proceedings of the 2nd AAAI Conference on Human Computation and Crowdsourcing.

Rafael M. Frongillo and Jens Witkowski. 2016. A geometric method to construct minimal peer prediction mechanisms. In Proceedings of the AAAI Conference on Artificial Intelligence (AAAI'16). 502-508.

A. Gao, J. R. Wright, and K. Leyton-Brown. 2016. Incentivizing evaluation via limited access to ground truth: Peer-prediction makes things worse. ArXiv e-prints. arxiv:cs.GT/1606.07042

Tilmann Gneiting and Adrian E. Raftery. 2007. Strictly proper scoring rules, prediction, and estimation. F. Amer. Statist. Assoc. 102, 477 (2007), 359-378.

Harold V. Henderson and Shayle R. Searle. 1981. The vec-permutation matrix, the vec operator and Kronecker products: A review. Linear Multilinear Algebra 9, 4 (1981), 271-288.

Radu Jurca and Boi Faltings. 2007. Collusion-resistant, incentive-compatible feedback payments. In Proceedings of the 8th ACM Conference on Electronic Commerce. ACM, 200-209.

Radu Jurca and Boi Faltings. 2009. Mechanisms for making crowds truthful. J. Artif. Int. Res. 34, 1 (Mar. 2009).

Vijay Kamble, Nihar Shah, David Marn, Abhay Parekh, and Kannan Ramachandran. 2015. Truth serums for massively crowdsourced evaluation tasks. arXiv preprint arXiv:1507.07045 (2015).

Yuqing Kong, Katrina Ligett, and Grant Schoenebeck. 2016. Putting peer prediction under the micro (economic) scope and making truth-telling focal. In Proceedings of the International Conference on Web and Internet Economics. Springer, 251-264.

Y. Kong and G. Schoenebeck. 2016. A framework for designing information elicitation mechanisms that reward truthtelling. ArXiv e-prints. arxiv:cs.GT/1605.01021

Yuqing Kong and Grant Schoenebeck. 2018b. Eliciting expertise without verification. In Proceedings of the ACM Conference on Economics and Computation. ACM, 195-212.

Yuqing Kong and Grant Schoenebeck. 2018a. Equilibrium selection in information elicitation without verification via information monotonicity. In Proceedings of the 9th Conference on Innovations in Theoretical Computer Science (ITCS'18), Vol. 94.

Yuqing Kong and Grant Schoenebeck. 2018c. Water from two rocks: Maximizing the mutual information. In Proceedings of the ACM Conference on Economics and Computation. ACM, 177-194. 
Friedrich Liese and Igor Vajda. 2006. On divergences and informations in statistics and information theory. IEEE Trans. Info. Theory 52, 10 (2006), 4394-4412.

Yang Liu and Yiling Chen. 2017. Machine-learning aided peer prediction. In Proceedings of the ACM Conference on Economics and Computation (EC'17). ACM, New York, NY, 63-80. DOI : https://doi.org/10.1145/3033274.3085126

Yang Liu and Yiling Chen. 2018. Surrogate scoring rules and a dominant truth serum for information elicitation. CoRR abs/1802.09158 (2018). arxiv:1802.09158. Retrieved from http://arxiv.org/abs/1802.09158.

Debmalya Mandal, Matthew Leifer, David C. Parkes, Galen Pickard, and Victor Shnayder. 2016. Peer prediction with heterogeneous tasks. arXiv preprint arXiv:1612.00928 (2016).

N. Miller, P. Resnick, and R. Zeckhauser. 2005. Eliciting informative feedback: The peer-prediction method. Manage. Sci. 51, 9 (2005), 1359-1373.

D. Prelec. 2004. A Bayesian truth serum for subjective data. Science 306, 5695 (2004), 462-466.

Goran Radanovic and Boi Faltings. 2013. A robust Bayesian truth serum for non-binary signals. In Proceedings of the 27th AAAI Conference on Artificial Intelligence (AAAI'13). 833-839.

Goran Radanovic and Boi Faltings. 2014. Incentives for truthful information elicitation of continuous signals. In Proceedings of the 28th AAAI Conference on Artificial Intelligence (AAAI'14).

Goran Radanovic and Boi Faltings. 2015. Incentive schemes for participatory sensing. In Proceedings of the International Conference on Autonomous Agents and Multiagent Systems. International Foundation for Autonomous Agents and Multiagent Systems, 1081-1089.

Blake Riley. 2014. Minimum truth serums with optional predictions. In Proceedings of the 4th Workshop on Social Computing and User Generated Content (SC'14).

William E. Roth. 1934. On direct product matrices. Bull. Amer. Math. Soc. 40, 6 (1934), 461-468.

Victor Shnayder, Arpit Agarwal, Rafael Frongillo, and David C. Parkes. 2016. Informed truthfulness in multi-task peer prediction. In Proceedings of the 2016 ACM Conference on Economics and Computation (EC'16). ACM, New York, NY, 179-196. DOI : https://doi.org/10.1145/2940716.2940790

Robert L. Winkler. 1969. Scoring rules and the evaluation of probability assessors. J. Amer. Statist. Assoc. 64, 327 (1969), 1073-1078.

Jens Witkowski. 2014. Robust Peer Prediction Mechanisms. Ph.D. Dissertation. Department of Computer Science, AlbertLudwigs-Universität Freiburg.

Jens Witkowski, Pavel Atanasov, Lyle H. Ungar, and Andreas Krause. 2017. Proper proxy scoring rules. In Proceedings of the AAAI Conference on Artificial Intelligence (AAAI'17). 743-749.

J. Witkowski and D. Parkes. 2012. A robust Bayesian truth serum for small populations. In Proceedings of the 26th AAAI Conference on Artificial Intelligence (AAAI'12).

Peter Zhang and Yiling Chen. 2014. Elicitability and knowledge-free elicitation with peer prediction. In Proceedings of the International Conference on Autonomous Agents and Multi-agent Systems. International Foundation for Autonomous Agents and Multiagent Systems, 245-252.

Received January 2018; revised June 2018; accepted October 2018 